\title{
Model-based process design of a ternary protein separation using multi-step gradient ion-exchange SMB chromatography
}

\author{
Qiao-Le He ${ }^{1,2}$ and Liming Zhao ${ }^{1,2}$ \\ ${ }^{1}$ State Key Laboratory of Bioreactor Engineering, East China University of Science and \\ Technology, 200237 Shanghai, China \\ ${ }^{2} R \& D$ Center of Separation and Extraction Technology in Fermentation Industry, East \\ China University of Science and Technology, 200237 Shanghai, China
}

Apr. 15, 2020

\begin{abstract}
Prominent features of simulated moving bed (SMB) chromatography processes in the downstream processing is based on the determination of operating conditions. However, effects of different types of uncertainties have to be studied and analysed whenever the triangle theory or numerical optimization approaches are applied. In this study, a Bayesian inference based method is introduced to consider the uncertainty of operating conditions on the performance assessment, of a glucose-fructose SMB unit under linear condition. A multiple chain Markov Chain Monte Carlo (MCMC) algorithm (i.e., Metropolis algorithm with delayed rejection and adjusted Metropolis) is applied to generate samples. The proposed method renders versatile information by constructing from the MCMC samples, e.g., posterior distributions, uncertainties, credible intervals of the operating conditions, and posterior predictive check, and Pareto fronts between each pair of the performance indicators. Additionally, the MCMC samples can be mapped onto the $\left(m_{\mathrm{II}}, m_{\mathrm{III}}\right)$ and $\left(m_{\mathrm{IV}}, m_{\mathrm{I}}\right)$ planes to show the actually complete separation region under uncertainties. The proposed method is a convenient tool to find both optimal values and uncertainties of the operating conditions. Moreover, it is not limited to SMB processes under the linear isotherm; and it should be more powerful in the nonlinear scenarios.
\end{abstract}

\section{Introduction}

Purification and separation are major concerns in the downstream processing of industries (Carta \& Jungbauer, 2010; Scopes, 2013). Chromatography is a prevailing purification and separation technology (Guiochon, 2002). Simulated moving bed (SMB) (Broughton \& Gerhold, 1961) as a continuous chromatographic separation technology is an alternative to the conventional batch chromatography, since SMB processes have characteristic features (e.g., high productivity and low solvent consumption) (Seidel-Morgenstern et al., 2008; Rajendran et al., 2009). It has been widely applied to separations of fine petrol-chemicals, sugars, and pharmaceuticals (Juza et al., 2000; Rajendran et al., 2009; Faria \& Rodrigues, 2015).

There are three types of issues in process designs of SMB systems. The first type deals with the determination of network configuration (e.g., total columns and optimum number of columns in individual zones), the geometry of columns (e.g., length, diameter, size distribution of beads). The second type arises in measurement of experimental parameters (e.g., axial dispersion coefficient, porosities, dead volumes and adsorption parameters). Optimization of operating conditions (e.g., zonal flowrates, switching time), with respect to studied objectives, appears in the third type. In designs of SMB separations, the key issue is the determination of the optimum values of operating parameters, that is, assuming that the network configuration, the column geometry and the packing parameters have been fixed, that the adsorption equilibrium properties of the components have been known from the column model calibration. 
Classical algebraic methods to the selection of operating conditions are McCabe-Thiele diagram (Ruthven \& Ching, 1989), the safety margin approach (Mihlbachler et al., 2001), the standing wave design (Ma \& Wang, 1997; Mallmann et al., 1998), and the triangle theory (Storti et al., 1993, 1995). The safety margin approach is, in principle, equivalent to the triangle theory, which is derived based on the equilibrium theory model (i.e., ideal column model with the linear isotherm). Initially, the triangle theory was barely suitable for determining zonal flowrates of SMB processes under the linear isotherm condition. It was later extended to processes described by Langmuir pattern nonlinear isotherms (Mazzotti et al., 1997). Then, versatile results on triangle theory for the design of SMB processes have been published (Nowak et al., 2012; Kim et al., 2016; Lim, 2004; Kazi et al., 2012; Bentley \& Kawajiri, 2013; Bentley et al., 2014; Sreedhar et al., 2014; Toumi et al., 2007; Silva et al., 2015; Kiwala et al., 2016). No research has yet been reported to extend the triangle theory to nonlinear isotherms, such as spreading model (Ghosh et al., 2013, 2014), steric mass-action (Brooks \& Cramer, 1992). The McCabe-Thiele diagram can be applied to processes described by any kind of adsorption isotherms, but is limited to binary separations. The standing wave design method that was originally suitable for linear and ideal SMB processes (Ma \& Wang, 1997) was extended to nonlinear and nonideal processes by considering the axial dispersion effects and mass transfer resistances with correlation terms to the design equations (?). Further, the effects of dead volumes and pressure drops that are vital in real applications were taken into account (?).

Although the above algebraic solutions are powerful tools for determination of operating conditions of SMB processes, more insights should be shed into the interpretation of rate-limiting mass transfer of the most current macro-molecules. Moreover, substances separated by SMB processes have recently been evolved from monosaccharides to macro proteins, which can undergo conformation, orientation changes and aggregation in the process. The thermodynamics of macro-molecules, as observed both in experiments (Clark et al., 2007) and molecular dynamics (Dismer \& Hubbuch, 2010; Liang et al., 2012; Lang et al., 2015), are much more complicated than that described by the linear isotherm, even by the Langmuir pattern or the steric massaction model. Therefore, finding operating conditions that guarantee the required performance indicators under nonlinear and nonideal conditions is a major challenge in SMB systems.

Numerical solutions can be an alternative to the theoretical algebraic solutions, to constitute the chromatograms of various network configurations, to calculate the performance indicators, to search for the optimum flowrates and switching time (Rodrigues et al., 2007; Agrawal \& Kawajiri, 2012; Nowak et al., 2012; Wu et al., 2013; Li et al., 2014a,b; Bentley et al., 2014; Sreedhar et al., 2014; Yao et al., 2015; Yu et al., 2015). Numerical solutions of different column models with various adsorption isotherms have been investigated (Dünnebier et al., 2000). The characteristic features of SMB processes can be further improved by systematically tuning the column configurations and operation modes (Zhang et al., 2002). However, process designs of SMB units in industries do not fully benefit from the model-based approaches (Briskot et al., 2019). This is mainly attributed to the lack of uncertainty analysis in process designs of SMB systems (Kurup et al., 2008; Borg et al., 2013).

In order to efficiently model, design, and control SMB processes, any type of uncertainties (e.g., from equipment calibration, signal detectors, model assumption, model calibration, numerical solution) that could affect the performance assessment should be taken into account. Without consideration or inaccurate estimation of dead volumes results in errors in the evaluated adsorption parameters (Samuelsson et al., 2008a; Grosfils et al., 2010) and in the selection of adsorption model by chromatogram fitting (Samuelsson et al., 2008b). The effects of concentration measurement error (Joshi et al., 2006) and detector disturbance (Zhang et al., 2001) on the estimation of adsorption parameters have been studied. Borg et al. (2013) analysed the quantitative effect of uncertainty in the experimental conditions (Monte Carlo sampled experimental conditions) on the parameter estimation of a chromatographic column model. Kurup et al. (2008) presented a Monte Carlo based uncertainty analysis method, to investigate the propagation of errors in the estimation of adsorption parameters on the performance variability of a glucose-fructose SMB system. Since discrepancies between simulation and experimental results have often been observed in the SMB field, it is necessary to embrace a method that inherently considers uncertainties.

In this study, Bayesian inference will be used to investigate the uncertainty of operating conditions of a glucose-fructose SMB system on the multiple performance indicators. Formulation of Bayes theorem into the 
process design of SMB processes will firstly be presented. The glucose-fructose case study is chosen such that cross-validation with the algebraic solution can be implemented. Bayesian inference allows for measurements of uncertainties in a system to be propagated in a mathematically consistent manner. Bayesian inference differs with the commonly adopted frequentist methods in the chromatographic field (i.e., Fisher information based method and bootstrapping approach). The Monte Carlo based methods belong to the bootstrapping approach. Although large uncertainties of adsorption and experimental parameters have been reported, by using both bootstrap and Fisher information matrix, it is hardly presented that how the uncertainties of operating conditions affect the predictive behaviour of SMB models from the Bayesian perspective.

The operating conditions of the four-zone SMB unit will be optimized by a stochastic algorithm, Markov Chain Monte Carlo (MCMC), with respect to conflicting objectives (purity, yield as a demonstration and can be further generalized to other objectives). Pareto fronts will be calculated for illustrating the best compromises between each pair of the performance indicators. Unlike multi-objective optimization algorithms (e.g., non-dominated sorted genetic algorithm (Zhang et al., 2002), strength Pareto evolutionary algorithms) that try to eliminate all the non-dominated points during optimization, MCMC serves on the sampling purpose, which is interested in sampling the Pareto optimal points as well as those near Pareto optimal. For the sampling purpose, MCMC not only accepts proposals with better objective value, but also accepts moves heading to non-dominated points with certain probability. Since numerical solutions are not derived from the equilibrium theory (thus not limited to linear scenarios), they are capable to enumerate, in principle, all the infinite combinations of operating conditions to depict the complete separation region for nonlinear situations. By using the MCMC sampling, however, a coherent connection between numerical solutions and the triangle theory can be generated; the complete separation region of the triangle theory can be intrinsically sketched for the SMB processes described by any kind of adsorption isotherms.

\section{Theory}

\subsection{Column model}

The general rate model (GRM) that accounts for various levels of mass transfer resistance in phases (Guiochon et al., 2006) is used to describe the transport behaviour of components in the columns. Convection and axial dispersion in the bulk liquid are considered, as well as film mass transfer and pore diffusion in the porous beads:

$$
\begin{aligned}
\frac{\partial c_{i}^{j}}{\partial t} & =-u_{\mathrm{int}}^{j} \frac{\partial c_{i}^{j}}{\partial z}+D_{\mathrm{ax}}^{j} \frac{\partial^{2} c_{i}^{j}}{\partial z^{2}}-\frac{1-\varepsilon_{c}}{\varepsilon_{c}} \frac{3}{r_{p}} k_{f, i}^{j}\left(c_{i}^{j}-c_{p, i}^{j}\left(r=r_{p}\right)\right) \\
\frac{\partial c_{p, i}^{j}}{\partial t} & =D_{p, i}^{j}\left(\frac{\partial^{2} c_{p, i}^{j}}{\partial r^{2}}+\frac{2}{r} \frac{\partial c_{p, i}^{j}}{\partial r}\right)-\frac{1-\varepsilon_{p}}{\varepsilon_{p}} \frac{\partial q_{i}^{j}}{\partial t}
\end{aligned}
$$

In Eq. (1), $z \in[0, L]$ denotes the axial position where $L$ is the column length, while $r \in\left[0, r_{p}\right]$ denotes the radial position where $r_{p}$ is the particle radius. Furthermore, $c_{i}^{j}, c_{p, i}^{j}$ and $q_{i}^{j}$ denote the interstitial, stagnant and stationary phase concentrations of component $i \in\{1, \ldots, M\}$ in column $j \in\{1, \ldots, N\}$, respectively. $t$ is the time, $\varepsilon_{c}$ and $\varepsilon_{p}$ are the column and particle porosities, $u_{\mathrm{int}}^{j}$ interstitial velocity; $D_{\mathrm{ax}}^{j}$ is the axial dispersion coefficient, $D_{p, i}^{j}$ the effective pore diffusion coefficient, and $k_{f, i}^{j}$ the film mass transfer coefficient. At the column inlet and outlet, Danckwerts boundary conditions (Barber et al., 1998) are applied:

$$
\left\{\begin{array}{l}
\left.\frac{\partial c_{i}^{j}}{\partial z}\right|_{z=0}=\frac{u_{\mathrm{int}}^{j}}{D_{\mathrm{ax}}^{j}}\left(c_{i}^{j}(z=0)-c_{\mathrm{in}, i}^{j}\right) \\
\left.\frac{\partial c_{i}^{j}}{\partial z}\right|_{z=L}=0
\end{array}\right.
$$


where $c_{\mathrm{in}, i}^{j}$ is the inlet concentration of component $i$ in column $j$ (cf. Eq. (5)). The boundary conditions at the particle surface and centre are described by:

$$
\left\{\begin{array}{l}
\left.\frac{\partial c_{p, i}^{j}}{\partial r}\right|_{r=r_{p}}=\frac{k_{f, i}^{j}}{\varepsilon_{p} D_{p, i}^{j}}\left(c_{i}^{j}-c_{p, i}^{j}\left(r=r_{p}\right)\right) \\
\left.\frac{\partial c_{p, i}^{j}}{\partial r}\right|_{r=0}=0
\end{array}\right.
$$

When the adsorption equilibrium can be described by the linear isotherm, the concentration of each component in the mobile phase of column $j, c_{p, i}^{j}$, and in the particle phase of column $j, q_{i}^{j}$, are linearly correlated:

$$
q_{i}^{j}=H_{i} c_{p, i}^{j}
$$

\section{$2.2 \quad$ Node model}

In this study, two adjacent columns $(j, j+1)$ are connected via a node $j$. Therefore, the node $j$ is located at the downstream side of column $j$ and the upstream side of column $j+1$. Only one or none of feed (F), desorbent (D), raffinate (R), or extract (E) streams exists at a time for a node. The occasion that nodes are not connected to a port (i.e., in the interior of a zone) occurs when more columns than zones are present, such as, eight columns in a four-zone scheme. A circular SMB loop is closed when the column indices follows $\bmod (j, N)=1$ (i.e., by identifying column $j=N+1$ with column $j=1$ ). The inlet concentration of component $i$ in column $j+1$ is calculated from mass balance of the node $j$ :

$$
c_{\mathrm{in}, i}^{j+1}=\frac{c_{\mathrm{out}, i}^{j} Q^{j}+\delta_{i}^{j}}{Q^{j+1}}
$$

where $c_{\text {out }, i}^{j}=c_{i}^{j}(t, z=L)$ denotes the outlet concentration of component $i$ in column $j, Q^{j}=\varepsilon_{c} u_{\text {int }}^{j} \pi d_{c}^{2} / 4$ the zonal flowrates and $d_{c}$ the column diameter. The $\delta^{j}$ is determined by the current role of node $j$ (i.e., F, $\mathrm{D}, \mathrm{R}, \mathrm{E}$ or none):

$$
\delta_{i}^{j}=\left\{\begin{array}{cl}
c_{\mathrm{in}, \mathrm{i}}^{F} Q^{F} & \text { feed } \\
c_{\mathrm{in}, \mathrm{i}}^{D} Q^{D} & \text { desorbent } \\
-c_{\mathrm{out}, \mathrm{i}}^{j} Q^{R} & \text { raffinate } \\
-c_{\mathrm{out}, \mathrm{i}}^{j} Q^{E} & \text { extract } \\
0 & \text { none }
\end{array}\right.
$$

where $c_{\mathrm{in}, i}^{F}$ and $c_{\mathrm{in}, i}^{D}$ are the component concentrations at feed and desorbent ports, and $Q^{F}, Q^{D}, Q^{R}, Q^{E}$ the volumetric flowrates at the feed, desorbent, raffinate, and extract ports. Column shifting is implemented by periodically permuting $\delta$ each switching time $t_{s}$. From a mathematical point of view, the switching operation can be represented by a shifting of the initial and boundary conditions for the single column (Klatt et al., 2002).

\subsection{Performance indicators}

In this study, performance indicators are all defined in terms of components, $i \in\{1, \ldots, M\}$, withdrawn at a four-zone SMB node, $j \in\{E, R\}$, within one switching time $t_{s}$. The definitions of performance indicators are all based on concentration integrals $\bar{c}_{\text {out }, i}^{j}$ of component $i$ at node $j$ upon cyclic steady state (CSS), averaged over one switching time, $t_{s}$ :

$$
\bar{c}_{\mathrm{out}, i}^{j}=\frac{1}{t_{s}} \int_{t=(k-1) t_{s}}^{k t_{s}} c_{i}^{j}(t, z=L) \mathrm{d} t
$$


In Eq. (7), $k>k_{\mathrm{CSS}}$ denotes the switching number.

Purity, yield and productivity are commonly used performance indicators for process assessment. The purity of a component $i, \mathrm{Pu}_{i}^{j}$, is the average concentration of this component in the collected fraction of all components at port $j$, Eq. (8). The yield, $\mathrm{Y}_{i}^{j}$, is the ratio between the amount of the desired component $i$ in the collected fraction at port $j$ and the amount injected in the column through the feed port, Eq. (9). The productivity, $\operatorname{Pr}_{i}^{j}$, is the withdrawn mass of the component $i$ at port $j$ per collection time relative to the total volume of the utilized packed bed in all columns, Eq. (10).

$$
\begin{gathered}
\mathrm{Pu}_{i}^{j}=\frac{\bar{c}_{\mathrm{out}, i}^{j}}{\sum_{k=1}^{M} \bar{c}_{\mathrm{out}, k}^{j}} \\
\mathrm{Y}_{i}^{j}=\frac{Q^{j} \bar{c}_{\mathrm{out}, i}^{j}}{Q^{F} c_{\mathrm{in}, i}^{F}} \\
\operatorname{Pr}_{i}^{j}=\frac{Q^{j} \overline{\mathrm{c}}_{\mathrm{out}, i}^{j}}{\left(1-\varepsilon_{c}\right) V_{c} N}
\end{gathered}
$$

In addition, throughput, solvent consumption, pressure drops and cycle times can also be considered to assess performance.

\subsection{Multi-objective optimization}

Consider a SMB model with $M$ components and $n$ parameters, $\theta \in \mathbb{R}^{n}$. Generally, operating conditions of SMB processes are systematically optimized by numerical algorithms such that the above performance indicators $\left(\Psi=\left[\mathrm{Pu}_{i}^{j}, \mathrm{Y}_{i}^{j}, \operatorname{Pr}_{i}^{j}, \ldots\right]\right)$ are all maximized. However, there are trade-off relationships among the performance indicators. For example, maximizing $\mathrm{Pu}_{i}^{j}$ would result in low values of $\mathrm{Y}_{i}^{j}$ and $\mathrm{Pr}_{i}^{j}$, and vice versa. Therefore, multi-objective optimization is applied.

A set of objectives can be combined into a single objective by adding each objective a pre-multiplied weight (the weighted method (Marler \& Arora, 2010)), or keeping just one of the objectives and with the rest of the objectives constrained (the $\varepsilon$-constraint method (Chankong \& Haimes, 2008; Mavrotas, 2009)). The latter method is used in this work; as a demonstration, maximizing the yields of components at the corresponding withdrawn ports with their purities constrained to be larger than thresholds $\varepsilon_{i}^{j}$ :

$$
\begin{array}{ll}
\min & f(\theta)=-\sum_{j \in\{R, E\}} \mathrm{Y}_{i}^{j} \\
\text { s.t. } & \left\{\begin{array}{l}
c^{j}(\theta): \mathrm{Pu}_{i}^{j}-\varepsilon_{i}^{j} \geqslant 0 \\
\theta_{\min } \leqslant \theta \leqslant \theta_{\max }
\end{array}\right.
\end{array}
$$

The searching domain is bounded, $\left[\theta_{\min }, \theta_{\max }\right]$. Extension of Eq. (11) to ternary or quaternary objectives is straightforward. The inequalities $c^{j}(\theta), j \in\{R, E\}$ is lumped into the objective function using penalty terms in this study, such that it can be solved as a series of unconstrained minimization problems with increasing penalty factors, $d_{k}$ :

$$
\min \mathcal{H}\left(\theta ; d_{k}\right)=f(\theta)+d_{k} g(\theta)
$$

In Eq. (12), the penalty function is chosen as $g(\theta)=\sum_{j \in\{R, E\}}\left\|\min \left\{0, c^{j}(\theta)\right\}\right\|^{2}$.

\subsection{Bayesian inference}

In the Bayesian framework, inference conclusions are made in terms of probabilities, which is used as the fundamental measure of uncertainties. Four distributions are briefly introduced here and will be discussed in detail later: 
1. Prior distribution, $p(\theta)$, represents a population of possible parameter values. It is where we can express our knowledge about parameters on the inference. The prior distribution should include all possible values of $\theta$, but realistically the information about $\theta$ contained in the data will far outweigh reasonable probability distributions.

2. Likelihood function (also called sampling distribution), $p(\Psi \mid \theta)$, is a conditional distribution that describes the probability of a data set, $\Psi$, for given parameters $\theta$. It is typically formulated as a function of the model parameters, and contains all the information relative to the evaluation of statistical evidence. The sampling distribution plays a major role in Bayesian inference over the prior distribution.

3. Marginal distribution, $p(\Psi)$, is an integral of the joint distribution (i.e., the product of prior distribution and likelihood function) over the parameter space. It describes the probability of data set, $\Psi$, that is a constant independent of model and model parameters. However, the constant value is very hard to compute.

4. Posterior distribution, $p(\theta \mid \Psi)$, is a conditional distribution that describes the probability of the parameter set, $\theta$, given the data $\Psi$. We also refer to the posterior distribution as the target distribution. It contains the desired information on the sought parameters.

The above distributions are related to each other by the Bayes theorem (Gelman et al., 2014):

$$
p(\theta \mid \Psi)=\frac{p(\Psi \mid \theta) p(\theta)}{p(\Psi)}
$$

where $p(\Psi)=\int p(\Psi, \theta) \mathrm{d} \theta=\int p(\theta) p(\Psi \mid \theta) \mathrm{d} \theta$. The integral is very hard and computationally expensive to calculate for multi-dimensional distributions. Therefore, Bayesian inference is often realized by approximating the unnormalized posterior distribution (cf. Eq. 14) via sampling (i.e., MCMC). Technically, the MCMC sampling allows to calculate the constant value, $p(\Psi)$. However, this is pointless, as 1$)$ it is sufficient to sample from Eq. 14 and 2) integrating $p(\Psi)$ takes the similar computational effort with calculating the sought posterior distribution, $p(\theta \mid \Psi)$.

$$
p(\theta \mid \Psi) \propto p(\Psi \mid \theta) p(\theta)
$$

Different types of methods can be applied to solve the minimization of $\mathcal{H}\left(\theta ; d_{k}\right)$, such as deterministic methods and heuristic methods. Bayesian inference shall be adopted in this study. In order to use Bayesian inference, the $\mathcal{H}\left(\theta ; d_{k}\right)$ is further formulated as a likelihood function in the following exponential form:

$$
p(\Psi \mid \theta) \stackrel{\text { def }}{=} \exp \left(-\frac{1}{2} \mathcal{H}\left(\theta ; d_{k}\right)\right)
$$

\subsection{Markov Chain Monte Carlo}

Based on the Markov chain theory to generate chains, MCMC is able to sample from complicated distributions. The more states that are collected, the more closely the distribution of the samples matches the desired distribution. Various MCMC algorithms have been developed, which mainly differ in computational complexity, robustness, and speed of convergence.

\subsubsection{Metropolis algorithm}

Metropolis algorithm (Metropolis et al., 1953) is one of the blocking bricks. It is a random-walk algorithm with Gaussian proposal for sampling the operating parameters $\theta$. The algorithm proceeds as follows:

1. Initialize a starting point, $\theta^{0}$, for example, from the prior distribution; construct a covariance matrix, $\Sigma$, for the proposal distribution (Gaussian distribution in the present work).

2. For $k=1,2, \ldots$ : 
- Based on the previous sample, $\theta^{k}$, a candidate $\tilde{\theta}$ is drawn from the Gaussian proposal distribution, $\mathcal{N}\left(\theta^{k}, \Sigma\right)$.

- A ratio $\gamma\left(\tilde{\theta}, \theta^{k}\right)$ of posterior distributions of the candidate, $\tilde{\theta}$, and the previous sample, $\theta^{k}$, with respect to the desired target distribution is calculated:

$$
\begin{aligned}
\gamma\left(\tilde{\theta}, \theta^{k}\right) & =\frac{p(\tilde{\theta} \mid \Psi)}{p\left(\theta^{k} \mid \Psi\right)}=\frac{p(\Psi \mid \tilde{\theta}) p(\tilde{\theta})}{p\left(\Psi \mid \theta^{k}\right) p\left(\theta^{k}\right)} \\
& =\exp \left\{-\frac{1}{2}\left(\mathcal{H}\left(\tilde{\theta} ; d_{k}\right)-\mathcal{H}\left(\theta^{k} ; d_{k}\right)\right)\right\} \frac{p(\tilde{\theta})}{p\left(\theta^{k}\right)}
\end{aligned}
$$

- The candidate is conditionally accepted with the following probability, where the random number, $\beta$, is drawn from the uniform distribution on the interval $[0,1]$.

$$
\theta^{k+1}= \begin{cases}\tilde{\theta} & \beta \leqslant \min \left(1, \gamma\left(\tilde{\theta}, \theta^{k}\right)\right) \\ \theta^{k} & \text { otherwise }\end{cases}
$$

- The index $k$ is increased by one and the procedure is repeated until a stopping criterion is satisfied (e.g., a predefined number of samples is reached).

Eventually, a sequence of random samples whose distribution approximates the target density is obtained. In implementation, a portion of samples (e.g., $25 \%$ ) are discarded as burn-in to diminish the influence of the starting point, $\theta^{0}$. Though the Metropolis algorithm has simple and easy to implement features, it has low efficiency. In sampling, numerous candidates can be rejected, resulting slow convergence to the target distribution. Further, when one chain is trapped into a local mode, it might never converge to the target density. Hence, several enhancements have been proposed in the literature. In this study, an adaptive Metropolis strategy and a delayed rejection (Haario et al., 2006) are applied to alleviate the drawbacks.

\subsubsection{Adaptive Metropolis strategy}

The convergence of the Metropolis algorithm can be accelerated by adapting shape of the proposal distribution (i.e., Gaussian distribution in this study), as determined by the covariance matrix, $\Sigma$. Fisher information matrix can be a typical choice for the initial covariance matrix, $\Sigma_{0}$,

$$
\Sigma_{0}=\tilde{\sigma}_{0} V\left(S^{T} S\right)^{-1} V^{T}
$$

where the matrices $U, S$ and $V$ are the singular value decomposition of the Jacobian of the chromatography model, i.e., sensitivity of the chromatogram with respect to the parameters at $\theta^{0}$. $\tilde{\sigma}_{0}$ is a hyperparameter. Further details can be found in A. Another practical option is to run a pre-simulation beforehand and then calculate an approximated $\Sigma_{0}$ from the samples.

Initially, iterations of the Metropolis algorithm are performed with $\Sigma_{0}$ for a fixed and pre-defined number. Then, the covariance matrix $\Sigma$ is adapted in regular intervals, based on the history of the Markov chain

$$
\Sigma=c \operatorname{Cov}(\Theta)+\varepsilon_{a} I
$$

where $\Theta \in \mathbb{R}^{k \times n}$ is the single Markov chain at iteration $k$. I is the identity matrix, and $c$ is a scaling factor proposed by Gelman et al. (2014) to be $c=2.4^{2} / \sqrt{n}$. A small $\varepsilon_{a}>0$ prevents $\Sigma$ from becoming singular. The covariance matrix $\operatorname{Cov}(\Theta)$ is calculated by using all previously computed samples of the current Markov chain. 


\subsubsection{Delayed rejection}

The efficiency can be enhanced by delaying the rejection of candidates. Instead of discarding a proposal when $\gamma\left(\tilde{\theta}, \theta^{k}\right) \leqslant 1$ is satisfied, a next stage of the Metropolis algorithm is performed with a shrunken covariance matrix, $a \Sigma$. A shrinking factor of $a=0.1$ can be applied.

Delayed rejection is applied to increase the robustness and efficiency of the adaptive Metropolis strategy. The initial covariance matrix $\Sigma_{0}$ often can not be approximated correctly, which leads to a high rejection rate at the beginning. Consequently, this makes the adaption very slow since only few distinct points are available for estimating a better covariance matrix. In order to evolve the proposal covariance matrix to the structure of target density, more points need to be accepted, by taking another more cautious move from the starting point when the first move is rejected (possibly due to the inappropriate large scaling). Further details can be found in (Haario et al., 2006) and B.

\subsubsection{Convergence criteria and effective sample size}

Consider that the samples collected from $m$ multiple chains are denoted as $\Phi \in \mathbb{R}^{k \times n \times m}$; samples for an estimated parameter $\theta_{\ell}(\ell \in\{1, \ldots, n\})$ are labelled as $\Phi_{\varkappa, \iota}^{\ell},(\varkappa \in\{1, \ldots, k\}, \iota \in\{1, \ldots, m\})$. Stopping criteria are required to stop the MCMC simulations. A potential scale reduction factor (a.k.a. Gelman criteria (Gelman et al., 2014)) can be applied to $\Phi^{\ell}$ to assess convergence conditions. It is a square root of the ratio of sample variances,

$$
\widehat{R}_{\ell}=\sqrt{\frac{\widehat{\operatorname{var}}^{+}(\mathcal{W}, \mathcal{B})}{\mathcal{W}}}
$$

which is based on between- and within-chain variances:

$$
\begin{gathered}
\mathcal{B}=\frac{k}{m-1} \sum_{\iota=1}^{m}\left(\bar{\Phi}_{. \iota}^{\ell}-\bar{\Phi}_{. .}^{\ell}\right)^{2} \\
\mathcal{W}=\frac{1}{m} \sum_{r=1}^{n} s_{\iota}^{2}
\end{gathered}
$$

Note that the burn-in part has been discarded in length of the chains $k$ here. $\bar{\Phi}_{\cdot \iota}^{\ell}=\frac{1}{k} \sum_{\varkappa=1}^{k} \Phi_{\varkappa, \iota}^{\ell}$ is the mean value of the chain $\iota$. $\bar{\Phi}^{\ell}$. is the mean value of the mean vector of $m$ chains, $\frac{1}{m} \sum_{\iota=1}^{m} \bar{\Phi}_{. \iota}^{\ell}$. Thus, $\mathcal{B}$ defines the between-chain variance; $s_{\iota}^{2}$ denotes the within-chain variance of the chain $\iota$. The sample variance, $\widehat{\operatorname{var}}^{+}(\mathcal{W}, \mathcal{B})$, is estimated by a weighted average of $\mathcal{W}$ and $\mathcal{B}$, namely

$$
\widehat{\operatorname{var}}^{+}(\mathcal{W}, \mathcal{B})=\frac{k-1}{k} \mathcal{W}+\frac{1}{k} \mathcal{B}
$$

Upon convergence of the MCMC algorithm diagnosed by, for instance, the above criteria, the samples collected, since then, from the multiple chains can be mixed up to approximate the target distribution. The effective number of independent simulation draws (i.e., effective sample size) for any optimized parameter $\theta_{\ell}$ can be estimated from Eq. 24.

$$
n_{\mathrm{eff}}=\frac{m k}{1+2 \sum_{t=1}^{\infty} \rho_{t}}
$$

where $\rho_{t}$ is the autocorrelation of the mixed-up chain of the parameter $\theta_{\ell}$ at lag $t$; In practice, however, we barely have a finite simulation length, so the calculation has to be approximated. 
Table 1: Reference parameters for the separation of glucose and fructose on an 8-column sMB laboratory plant.

\begin{tabular}{cclcc}
\hline Catalogue & Symbol & Description & Value & Unit \\
\hline \multirow{4}{*}{$d_{c}$} & column diameter & $2.6 \cdot 10^{-2}$ & $\mathrm{~m}$ \\
& $d_{p}$ & particle diameter & $3.25 \cdot 10^{-3}$ & $\mathrm{~m}$ \\
& $\varepsilon$ & column void & 0.38 & \\
& $H_{i}$ & Henry constants & {$[0.28,0.54]$} & \\
& $c_{i}^{F}$ & Feed concentration & $3.05 \cdot 10^{3}$ & $\mathrm{~mol} \mathrm{~m}^{-3}$ \\
\hline \multirow{6}{*}{$\theta$} & column length & $5.36 \cdot 10^{-1}$ & $\mathrm{~m}$ \\
& $t_{s}$ & Switching time & $1.552 \cdot 10^{3}$ & $\mathrm{~s}$ \\
& $Q^{\text {rec }}$ & Recycle flowrate & $1.395 \cdot 10^{-7}$ & $\mathrm{~m}^{3} \mathrm{~s}^{-1}$ \\
& $Q^{F}$ & Feed flowrate & $2.00 \cdot 10^{-8}$ & $\mathrm{~m}^{3} \mathrm{~s}^{-1}$ \\
$Q^{R}$ & Raffinate flowrate & $2.66 \cdot 10^{-8}$ & $\mathrm{~m}^{3} \mathrm{~s}^{-1}$ \\
& $Q^{D}$ & Desorbent flowrate & $4.14 \cdot 10^{-8}$ & $\mathrm{~m}^{3} \mathrm{~s}^{-1}$ \\
$Q^{E}$ & Extract flowrate & $3.48 \cdot 10^{-8}$ & $\mathrm{~m}^{3} \mathrm{~s}^{-1}$ \\
\hline
\end{tabular}

\section{Case}

A monosaccharide mixture of glucose and fructose on a laboratory scale four-zone SMB process is used as a model example in this study, $i \in\{$ glc, fru $\}$ (Klatt et al., 2002). There are eight columns and two columns in each zone. The plant was reported to be operated at $60^{\circ} \mathrm{C}$. The liquid density can be considered as constant for the given feed concentration, and the adsorption isotherm is well-described by Henry's law. The parameters are classified into two catalogues, $[\varphi, \theta] ; \theta$ is treated as decision variables (i.e., degree of freedom) and shall be optimized in this study while $\varphi$, as experimental conditions, is kept unchanged and directly used, see Tab. 1.

In this study, all columns are assumed to be identical (e.g., packing density and porosities) and initially empty. Pure buffer is used as the desorbent, i.e., $c_{\mathrm{in}, i}^{D}=0 \mathrm{~mol} \mathrm{~m}^{-3}$. The feed concentrations are converted from $c_{\mathrm{in}, i}^{F}=550 \mathrm{gl}^{-1}$, assuming that fructose and glucose have the same molar mass of $180.16 \mathrm{~g} \mathrm{~mol}^{-1}$. Four typical axial dispersion coefficients $D_{\mathrm{ax}}^{j}, j \in\{\mathrm{I}, \mathrm{II}, \mathrm{III}, \mathrm{IV}\}$, are used since the values are not given in Klatt et al. (2002). The volumetric flowrates $Q^{j}$ in zones $j \in\{$ II, III, IV $\}$ are calculated from the recycle and F, D, $\mathrm{E}, \mathrm{R}$ flowrates. The interstitial velocities in zones are calculated from the respective volumetric flowrates, $u_{\text {int }}^{j}=Q^{j} /\left(\varepsilon_{c} A\right)$, where $A$ is the cross-section area.

The equilibrium-dispersive model (EDM) was used in Klatt et al. (2002), as the axial dispersion effects can not be neglected and the mass transfer are fast but not infinitely fast. Though the CADET is originally designed for solving the GRM, it can be adapted for solving the EDM as follows: Eq. 1 is spatially discretized using the finite volume method with only one radial cell, $N_{r}=1$ (the axial column dimension is discretized into $N_{z}=40$ cells). A very small particle porosity is used, $\varepsilon_{p}=10^{-5}$, such that the column porosity is asymptotic to the total porosity, $\lim _{\varepsilon_{p} \rightarrow 0} \varepsilon_{c}=\lim _{\varepsilon_{p} \rightarrow 0} \frac{\varepsilon_{t}-\varepsilon_{p}}{1-\varepsilon_{p}}=\varepsilon_{t}$. The effective pore diffusion coefficient and the film mass transfer coefficient are made sure not to be rate limiting, $D_{p}=5 \cdot 10^{-5} \mathrm{~m}^{2} \mathrm{~s}^{-1}$ and $k_{f}=1.6 \cdot 10^{4} \mathrm{~m} \mathrm{~s}^{-1}$.

The mathematical models described above for each column of the SMB processes are weakly coupled together and then iteratively solved. The open-source code has been published on Github, https://github . com/modsim/CADET-SMB. git. CADET-SMB repeatedly invokes CADET kernel to solve each individual column model. The resulting system of ordinary differential equations is solved using an absolute tolerance of $10^{-10}$, relative tolerance of $10^{-6}$, an initial step size of $10^{-14}$ and a maximal step size of $5 \cdot 10^{6}$. CADET is also an open-source software published on Github, https://github.com/modsim/CADET.git. All numerical simulations are computed on an $\operatorname{Intel}(\mathrm{R}) \mathrm{Xeon}(\mathrm{R})$ system with $16 \mathrm{CPU}$ cores (64 threads) running at $2.10 \mathrm{GHz}$.

A stochastic multi-objective sampling algorithm, MCMC, is applied in this study to optimize the operating conditions. The Metropolis algorithm, incorporating with delayed rejection and adjusted Metropolis, has been published as open-source software on Github, https://github.com/modsim/CADET-MCMC.git. Noninformative prior distribution $p(\theta)$ is used. Samples are collected until either the Gelman criteria for each 
Table 2: Boundary conditions of the operating parameters of the four-zone scheme.

\begin{tabular}{clccc}
\hline \multirow{2}{*}{ Symbol } & \multirow{2}{*}{ Description } & \multicolumn{2}{c}{ Value } & \multirow{2}{*}{ Unit } \\
\cline { 3 - 4 } & & $\min$ & $\max$ & \\
\hline$L$ & column length & $50.0 \cdot 10^{-2}$ & $60.0 \cdot 10^{-2}$ & $\mathrm{~m}$ \\
$t_{s}$ & switching time & $1.5 \cdot 10^{3}$ & $1.6 \cdot 10^{3}$ & $\mathrm{~s}$ \\
$Q^{\text {rec }}$ & recycle flowrate & $1.0 \cdot 10^{-7}$ & $1.8 \cdot 10^{-7}$ & $\mathrm{~m}^{3} \mathrm{~s}^{-1}$ \\
$Q^{F}$ & feed flowrate & $1.5 \cdot 10^{-8}$ & $2.5 \cdot 10^{-8}$ & $\mathrm{~m}^{3} \mathrm{~s}^{-1}$ \\
$Q^{D}$ & desorbent flowrate & $3.5 \cdot 10^{-8}$ & $4.5 \cdot 10^{-8}$ & $\mathrm{~m}^{3} \mathrm{~s}^{-1}$ \\
$Q^{E}$ & extract flowrate & $3.0 \cdot 10^{-8}$ & $4.0 \cdot 10^{-8}$ & $\mathrm{~m}^{3} \mathrm{~s}^{-1}$ \\
\hline
\end{tabular}

parameter, $\widehat{R}_{\ell}, \ell \in\{1, \ldots, n\}$, is smaller than 1.1 or the maximal sample size (i.e., 400) is reached. $\widehat{R}_{\ell}$ declines to 1 when $k \rightarrow \infty$; but we generally have been satisfied with setting 1.1 as a threshold. Smooth probability density of each marginal posterior distribution is estimated by a MATLAB routine, ksdensity. Multiple chains of MCMC are used, but not intrinsically. Two MCMC simulation instances (i.e., $m=2$ ) are run simultaneously on the computing node; meanwhile, samples of each chain are written into the shared memory of the node. An additional program of the convergence diagnose is running parallel on the node, accessing the convergence conditions periodically with the samples having been stored on the shared memory. The Pareto fronts in this study describe two-dimensional trade-offs between a pair of the performance indicators, $\Psi$. Thus, the non-dominated stable sort method of Pareto front is applied to generate the frontiers (Duh et al., 2012).

\section{Results and discussion}

\subsection{Multi-objective optimization}

Searching domain of the operating parameters, $\theta$, is listed in Tab. 2. The boundary intervals are based on the optimal condition of Klatt et al. (2002) with additional safety margins. The maximal sampling length of MCMC is $k=400$ with the burn-in length of 50. The autocorrelation plot for each parameter, $\theta_{\ell}, \ell \in\{1, \ldots, n\}$, is shown in the C. The Gelman criteria for the optimized parameters are $\widehat{R}=$ $[1.07,0.99,1.01,0.99,1.00,0.99]$ when $k=309$. As seen from the convergence diagnose (i.e., $\widehat{R}_{\ell}=1.07$ ), the parameter, column length, converges slower than the other parameters. The average effective sample size of all parameters upon convergence $n_{\mathrm{eff}}=78$ in this case. Therefore, no more than $n_{\mathrm{eff}}$ samples are required to approximate the target distribution. The sampling length and the effective sample size are rather small in the framework of Bayesian inference. This is partially because of that the initial point used for the MCMC sampling is a optimal point located in the stationary region of the multivariate posterior distribution; partially it is a four-zone SMB with the linear isotherm. If an initial point that is far away from the stationary region was used, it could exert significant impact on the efficiency and convergence of the MCMC algorithm.

As the glucose has lower value of Henry coefficient than fructose $H_{\text {glc }}<H_{\text {fru }}$ (thus lower retention time), it is collected at the raffinate port of the four-zone SMB process; while fructose is collected at the extract port. Fig. 1 shows the Pareto fronts between each pair of the performance indicators $\Psi=\left[\mathrm{Pu}_{i}^{j}, \mathrm{Y}_{i}^{j}, \operatorname{Pr}_{i}^{j}\right]$ considered in the case study. At the extract port, high yield of fructose $Y_{\text {fru }}^{E}$ can be achieved with a wide range of purity $\mathrm{Pu}_{\text {fru }}^{E}$ from $60 \%$ to $100 \%$ (Fig. 1a); While at the raffinate port, rather high purity of glucose, $\mathrm{Pu}_{\mathrm{glc}}^{R}=99.9 \%$, can be achieved within a wide range of yield, $\mathrm{Y}_{\mathrm{glc}}^{R}$ (Fig. 1b). It implies that the less adsorbed component, glucose, is inclined to spread to the extract port, resulting in low purity of fructose at the extract port, and low yield of glucose at the raffinate port. This shall be explained from another point of view in section 4.3. With resort to the four-zone SMB scheme, operating conditions that render high purities $\left[\mathrm{Pu}_{\mathrm{glc}}^{R}, \mathrm{Pu}_{\mathrm{fru}}^{E}\right]$ and yield $\left[\mathrm{Y}_{\mathrm{glc}}^{R}, \mathrm{Y}_{\mathrm{fru}}^{E}\right]$ at both outlet streams can be found (cf. Fig. 1c-1d). According to the definitions of yield and productivity, both indicators increase with amounts of the product collected. However, productivity can also be enhanced by reducing the switching time, $t_{s}$, that is optimized. At a rather high purity requirement of $\mathrm{Pu}_{\mathrm{fru}}^{E}=99.9 \%$, a productivity $\operatorname{Pr}_{\text {fru }}^{E}$ of ca. $4.0 \cdot 10^{-2} \mathrm{~mol} \mathrm{~m}^{-3} \mathrm{~s}^{-1}$ can be achieved at the extract port; while at purity of $80 \%$, the productivity increases to ca. $5.0 \cdot 10^{-2} \mathrm{~mol} \mathrm{~m}^{-3} \mathrm{~s}^{-1}$ (Fig. 1e). At the raffinate port, 
Table 3: Performance indicators of the three characteristic points on the Pareto fronts

\begin{tabular}{ccccccr}
\hline Point & $\mathrm{Pu}_{\mathrm{fru}}^{E}[\%]$ & $\mathrm{Pu}_{\mathrm{glc}}^{R}[\%]$ & $\mathrm{Y}_{\mathrm{fru}}^{E}$ & $\mathrm{Y}_{\mathrm{glc}}^{R}$ & $\operatorname{Pr}_{\mathrm{fru}}^{E}\left[\mathrm{~mol} \mathrm{~m}^{-3} \mathrm{~s}^{-1}\right]$ & $\operatorname{Pr}_{\mathrm{glc}}^{R}\left[\mathrm{~mol} \mathrm{~m}^{-3} \mathrm{~s}^{-1}\right]$ \\
\hline$a$ & 85.21 & 99.98 & 1.00 & 0.83 & $4.06 \cdot 10^{-2}$ & $3.35 \cdot 10^{-2}$ \\
$b$ & 99.92 & 99.92 & 1.00 & 1.00 & $3.74 \cdot 10^{-2}$ & $3.74 \cdot 10^{-2}$ \\
$c$ & 99.95 & 99.01 & 0.99 & 1.00 & $4.16 \cdot 10^{-2}$ & $4.21 \cdot 10^{-2}$ \\
\hline
\end{tabular}

high productivity of $\operatorname{Pr}_{\mathrm{glc}}^{R}=5.0 \cdot 10^{-2} \mathrm{~mol} \mathrm{~m}^{-3} \mathrm{~s}^{-1}$ at hight purity of $\mathrm{Pu}_{\mathrm{glc}}^{R}=99.9 \%$ can be achieved (Fig 1f).

The Pareto fronts provide full trade-off information of the four-zone SMB scheme for separating glucose and fructose. Three characteristic points on the Pareto frontiers, as listed in Tab. 3, are exemplified. Points, $a, b, c$, were chosen on purpose; $\mathrm{Pu}_{\mathrm{fru}}^{E}$ is increasing while $\mathrm{Pu}_{\text {glc }}^{R}$ is decreasing. On the language of triangle theory, operating points are picked from the pure raffinate region $(a)$, the complete separation region $(b)$ and pure extract region $(c)$, respectively. The corresponding chromatograms along the four-zone SMB train are shown in Fig. 2. As stated in section 2.4, the multi-objective optimization can be straightforwardly generalize to other performance indicators, beyond the demonstration of yield and purity here.

\subsection{Uncertainty of operating conditions}

Marginal posterior distributions of the operating conditions listed in Tab. 2 were generated directly from the collected MCMC samples; while the distributions of $Q^{R}, Q^{\mathrm{II}}, Q^{\mathrm{II}}, Q^{\mathrm{IV}}$ were calculated with the following vector operations:

$$
\begin{aligned}
Q^{\mathrm{II}} & =Q^{\mathrm{rec}}-Q^{E} \\
Q^{\mathrm{III}} & =Q^{\mathrm{II}}+Q^{F} \\
Q^{\mathrm{IV}} & =Q^{\mathrm{rec}}-Q^{D} \\
Q^{R} & =Q^{D}+Q^{F}-Q^{E}
\end{aligned}
$$

Fig. 3a-3j show the marginal posterior distributions, resulting in the $\mathrm{Y}_{i}^{j}=1, \mathrm{Pu}_{i}^{j} \geqslant 99.9 \%$ target. They are all unimodal. The widths of the unimodal distributions indicate that the operating conditions are well-determined on the parameter estimation point of view.

For the flowrates of recycle, $Q^{\text {rec }}$, feed, $Q^{F}$ and raffinate, $Q^{R}$, they have distributions of smooth Gaussian shapes; while for the rest parameters, they are asymmetric (e.g., Fig. 3e, 3i), skewed (e.g., Fig. 3h, 3j) and tailing (e.g., Fig. 3b, 3f). In other words, they might be rough in shapes. The roughness could potentially be polished by running the MCMC simulations much longer such that each convergence diagnostics goes to 1 (i.e., $k \rightarrow \infty, \widehat{R}_{\ell} \rightarrow 1$ ). But, the roughness does not substantially disturb to make inference here, as the convergence criteria are satisfied with rather small thresholds and samples larger than the effective sample size have been collected. Roughness of distribution shapes and computational consumption are under a trade-off relationship. In SMB, CSS can be obtained only in an asymptotic sense, which means that it will take a large number of periods, hence a long time to enter into it. It takes $80 \sim 144$ iterations (i.e., $1.7 \sim 3.5 \mathrm{~h}$ on the computing node) for each SMB simulation to converge to CSS with tolerance criterion of $10^{-5}$. Longer sampling length of MCMC results in large computational burden.

The blue lines in Fig. 3 are the parameter values from Klatt et al. (2002). As shown, the column length increases from $0.56 \mathrm{~m}$ to $0.57 \mathrm{~m}$, correspondingly, the switching time increases from $1552 \mathrm{~s}$ to $1580 \mathrm{~s}$. The volumetric flowrates at feed and extract ports reduce, while all the other flowrates increase. For comparison, the parameter sets of points $(a, b, c)$ are also marked with coloured lines.

The distribution results are further analysed by credible intervals $(\mathrm{CI}),[\breve{\delta}, \hat{\delta}]$, of each parameter $\theta_{\ell}$ (cf. Eq. (26)). It is approximated by taking the $\alpha / 2$ and $1-\alpha / 2$ percentiles from the collected samples 


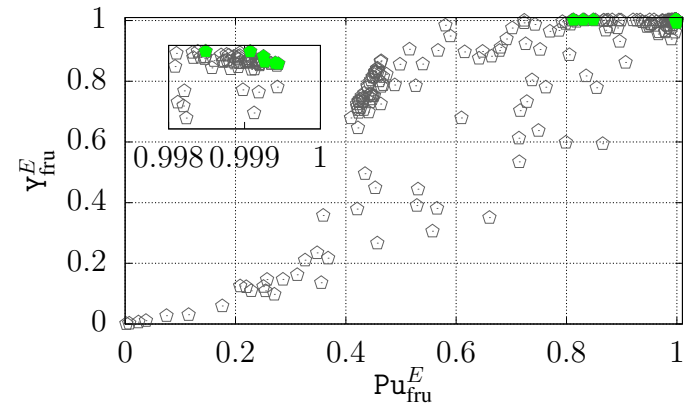

(a)

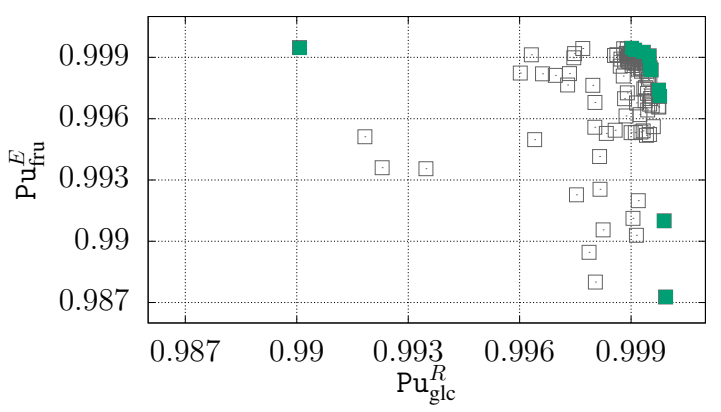

(c)

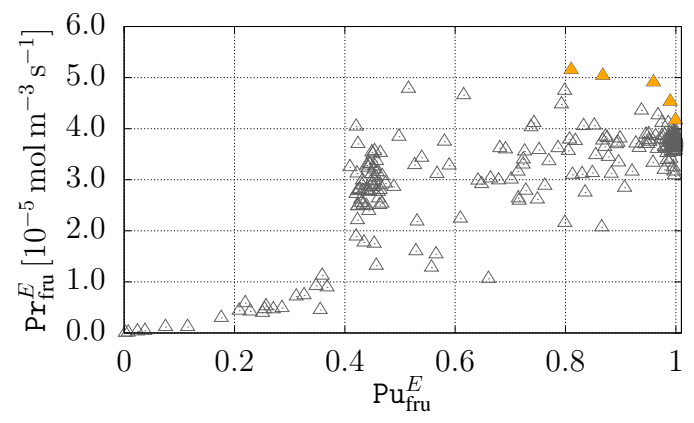

(e)

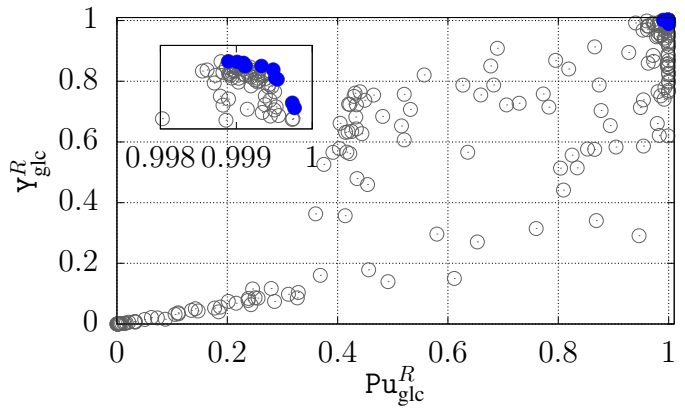

(b)

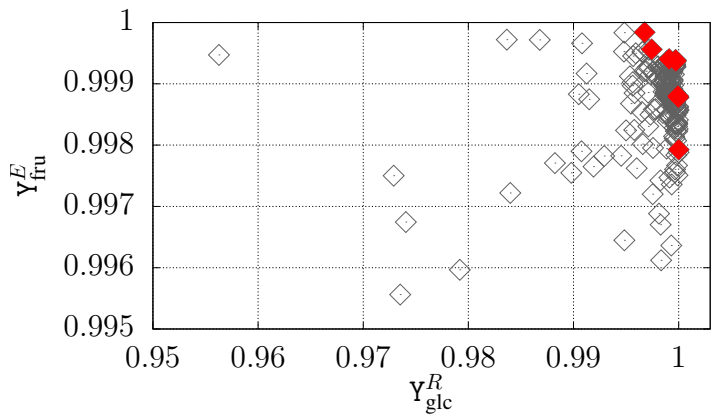

(d)

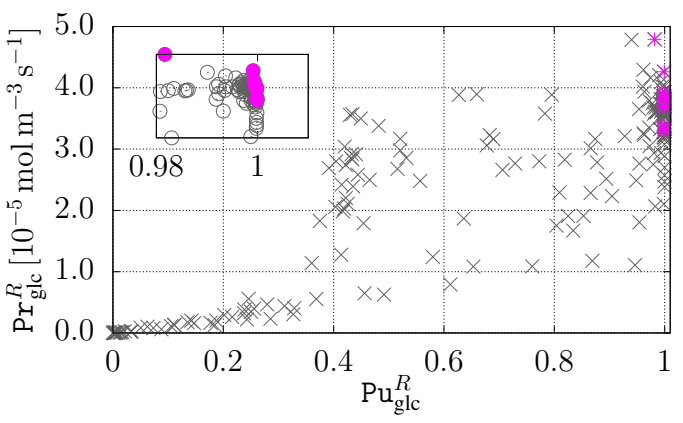

(f)

Figure 1: Pareto fronts between each pair of performance indicators $\Psi=\left[\mathrm{Pu}_{i}^{j}, \mathrm{Y}_{i}^{j}, \operatorname{Pr}_{i}^{j}\right], i \in\{\mathrm{glc}$, fru $\}, j \in$ $\{R, E\}$. The coloured symbols illustrate the Pareto fronts, while the gray symbols illustrate samples from MCMC and show the convergence trajectories. $a, b, c$ are three points chosen for exemplification. 


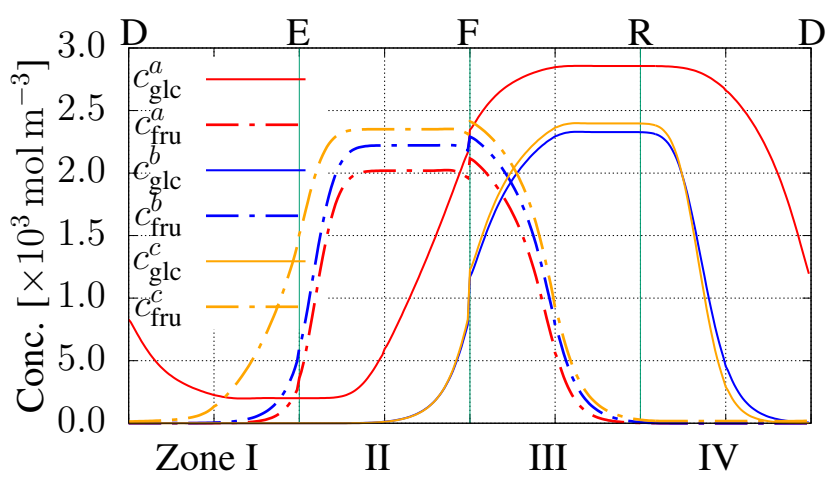

Figure 2: Chromatograms of the characteristic points $(a, b, c)$ on the Pareto fronts. D, E, F, R denote desorbent, extract, feed and raffinate respectively.

Table 4: Credible intervals of the operating conditions of the four-zone smB process.

\begin{tabular}{clll|l|l}
\hline$\theta$ & $\mu$ & $\breve{\delta}$ & $\hat{\delta}$ & $\frac{\mu-\breve{\delta}}{\mu}\left|\frac{\mu-\hat{\delta}}{\mu}\right| \%$ \\
\hline$L$ & 0.569 & 0.566 & 0.573 & 0.5 & 0.7 \\
$t_{s}$ & $1.577 \cdot 10^{3}$ & $1.558 \cdot 10^{3}$ & $1.592 \cdot 10^{3}$ & 1.2 & 1.0 \\
$Q^{\text {rec }}$ & $1.450 \cdot 10^{-7}$ & $1.430 \cdot 10^{-7}$ & $1.461 \cdot 10^{-7}$ & 1.4 & 2.1 \\
$Q^{F}$ & $1.773 \cdot 10^{-8}$ & $1.669 \cdot 10^{-8}$ & $1.834 \cdot 10^{-8}$ & 5.9 & 3.4 \\
$Q^{D}$ & $4.482 \cdot 10^{-8}$ & $4.420 \cdot 10^{-8}$ & $4.491 \cdot 10^{-8}$ & 1.4 & 0.2 \\
$Q^{E}$ & $3.203 \cdot 10^{-8}$ & $3.157 \cdot 10^{-8}$ & $3.288 \cdot 10^{-8}$ & 1.4 & 2.7 \\
$Q^{R}$ & $2.988 \cdot 10^{-8}$ & $2.951 \cdot 10^{-8}$ & $3.069 \cdot 10^{-8}$ & 1.2 & 2.7 \\
$Q^{\mathrm{II}}$ & $1.117 \cdot 10^{-7}$ & $1.110 \cdot 10^{-7}$ & $1.136 \cdot 10^{-7}$ & 0.6 & 1.7 \\
$Q^{\mathrm{III}}$ & $1.308 \cdot 10^{-7}$ & $1.277 \cdot 10^{-7}$ & $1.317 \cdot 10^{-7}$ & 2.4 & 0.7 \\
$Q^{\mathrm{IV}}$ & $1.006 \cdot 10^{-7}$ & $9.805 \cdot 10^{-8}$ & $1.018 \cdot 10^{-7}$ & 2.5 & 1.2 \\
\hline
\end{tabular}

while ignoring all other entries in the parameter vector.

$$
\begin{aligned}
\int_{\mathrm{CI}} p\left(\theta_{\ell} \mid y\right) \mathrm{d} \theta_{\ell} & =1-\alpha, \quad 0<\alpha<1 \\
p\left(\theta_{\ell} \mid y\right) & =\int \ldots \int p(\theta \mid y) \mathrm{d}\left(\theta_{1}, \ldots, \theta_{\ell-1}, \theta_{\ell+1}, \ldots, \theta_{n}\right)
\end{aligned}
$$

CI in the framework of Bayesian inference is equivalent to the confidence intervals in the frequentist statistics. In Tab. 4, the CI of operating conditions and the values with maximal posterior probabilities, $\mu$, are shown. In this study, $1-\alpha=0.66$, that is, $66 \%$ credible intervals. The credible intervals are, in majority, within $5 \%$ deviation of $\mu$. Therefore, the operating conditions are well-determined.

Posterior distributions of parameters can also be used to interpret the robustness of smB processes, by means of posterior predictive check (PPC) in the framework of Bayesian inference. Only uncertainty of operating conditions are take into consideration in this study. The effects caused by model selection, model and experimental calibrations have not been taken into account, such as feed concentration and composition, and poor estimation of kinetic parameters in the model calibration. Fig. 4 shows the PPC of resulting posterior distributions. 30 random sample from the posterior distributions were used to do forward simulation of the SMB process. Maximal and minimal chromatogram values at each observation point along the SMB train were recorded. In Fig. 4, the light blue filled curves indicate the deviation region of the chromatogram of glucose; while the light green ones show the deviation region of the chromatogram of fructose. The SMB process is robust, as it has tolerance to perturbations in the deviations of the posterior distributions (cf. Fig. 3), though the highest concentration values vary. PPC should be adopted as the chromatograms for experimental data to compare, rather than a single chromatogram from a single set of operating condition. 


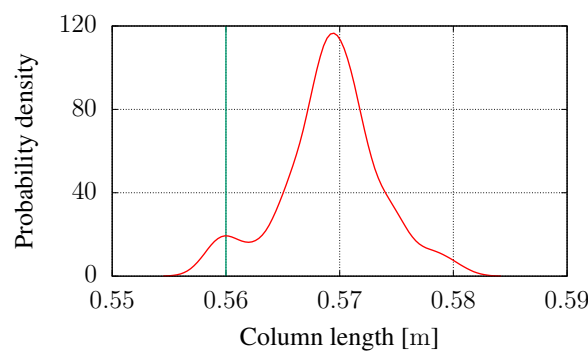

(a) Column length

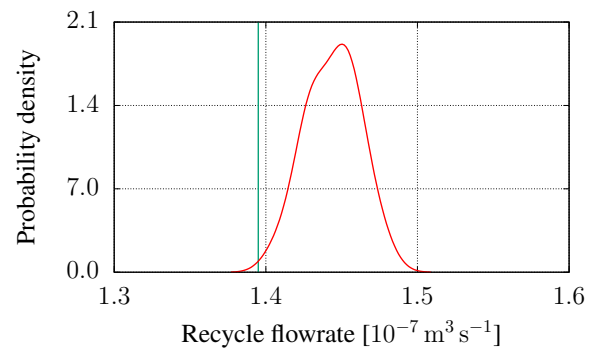

(c) Recycle flowrate

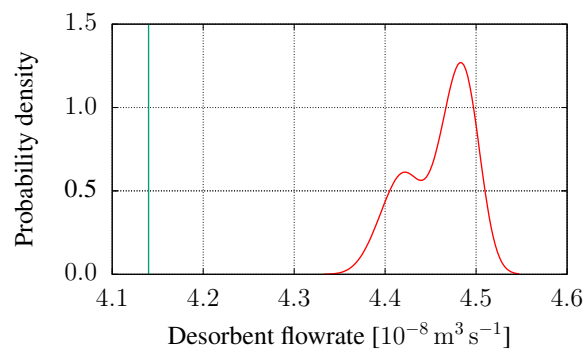

(e) Desorbent flowrate

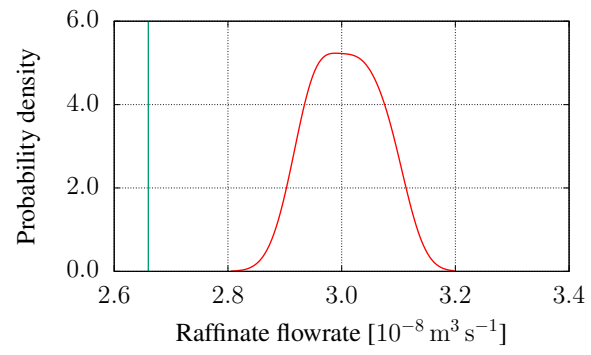

(g) Raffinate flowrate

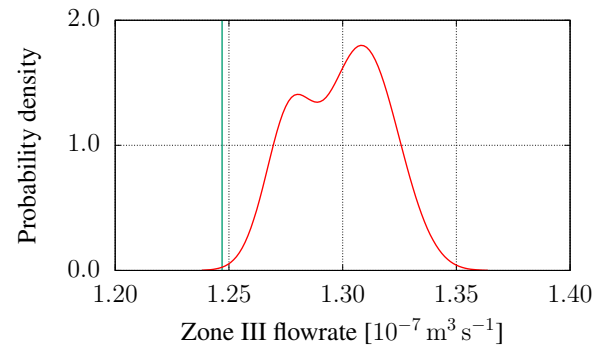

(i) Zone III flowrate

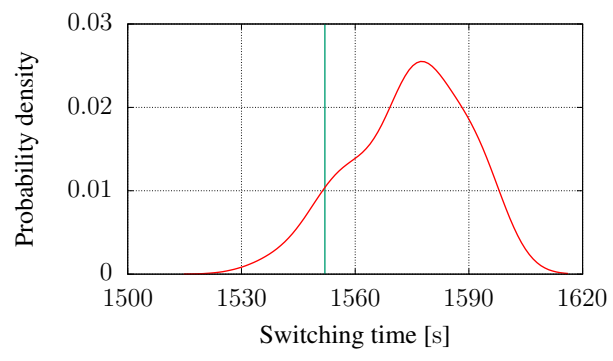

(b) Switching time

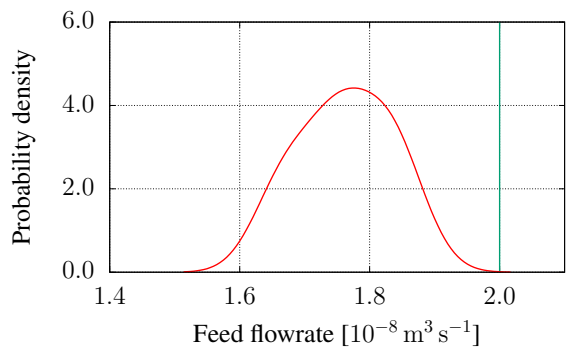

(d) Feed flowrate

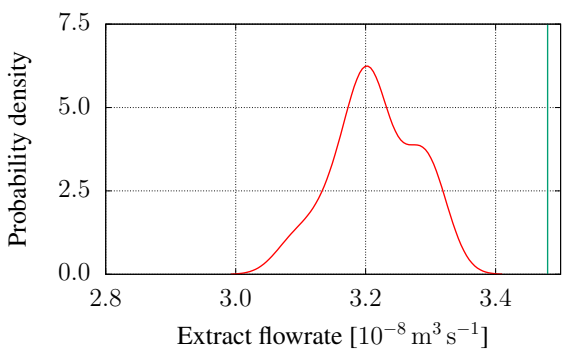

(f) Extract flowrate

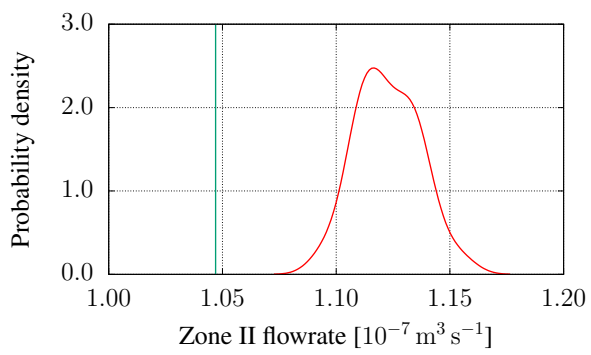

(h) Zone II flowrate

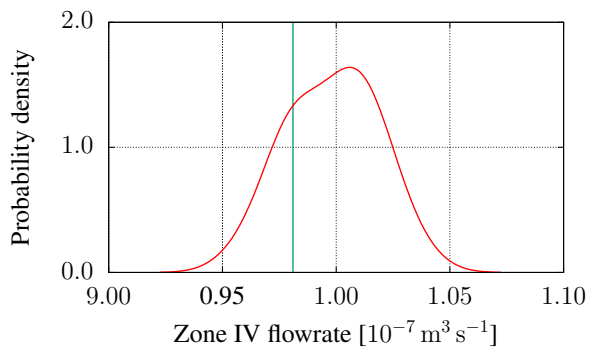

(j) Zone IV flowrate

Figure 3: Posterior distributions of the operating conditions of the four-zone SMB process, which result in the $\mathrm{Y}_{i}^{j}=1, \mathrm{Pu}_{i}^{j} \geqslant 99.9 \%$ target. The blue lines denote the parameter values from Klatt et al. (2002); purple lines for the point $a$, black lines for the point $b$, yellow lines for the point $c$. 


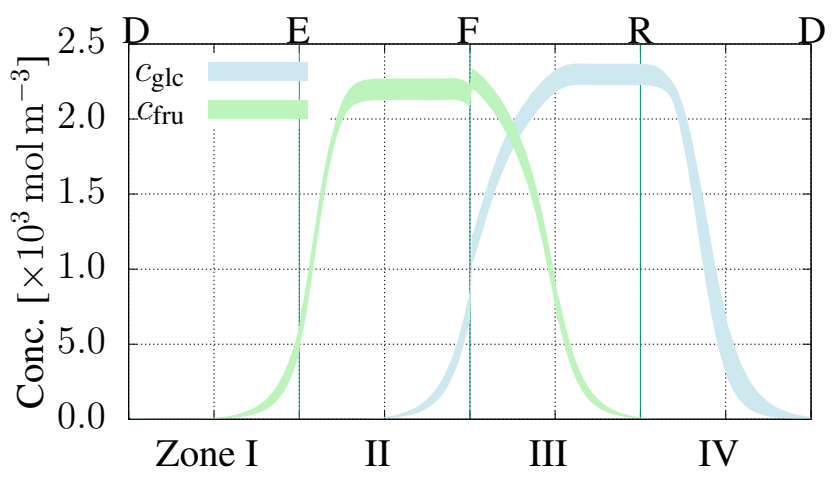

Figure 4: Posterior predictive check with the MCMC samples from the stationary region. D, E, F, R denote desorbent, extract, feed and raffinate respectively.

Therefore, by considering uncertainties in process design and PPC of posterior distributions, discrepancies between experimental and simulated data can be explained.

\subsection{Mapping to the triangle theory}

We are interested in determining the optimal operating conditions, $\theta$, of the four-zone SMB process, for a given feed composition (i.e., $c_{\mathrm{in}, i}^{F}=550 \mathrm{~g} \mathrm{l}^{-1}=3.0528 \cdot 10^{3} \mathrm{~mol} \mathrm{~m}^{-3}, i \in\{$ glc, fru $\}$ ) in this study. By using the triangle theory (provided the feed concentration is diluted such that nonlinear effects are negligible), the space of the operating parameters (i.e., the dimensionless flowrate ratios, $m_{j}, j \in\{\mathrm{II}, \mathrm{III}\}$ ) is divided into four major regions with different separation regimes (illustrated as $\mathcal{A}, \mathcal{B}, \mathcal{C}, \mathcal{D}$ in Fig. 5a). The boundaries (i.e., $w x, w y$ ) of the complete separation region, $\mathcal{A}$, are calculated by Eq. (27), which involves the adsorption parameters of glucose and fructose, $H_{\text {glc }}$ and $H_{\text {fru }}$.

$$
\begin{aligned}
H_{\mathrm{glc}} & <m_{\mathrm{II}}<m_{\mathrm{III}}<H_{\text {fru }} \\
m_{j} & =\frac{t_{s} Q^{j}-\varepsilon_{t} V_{c}}{\left(1-\varepsilon_{t}\right) V_{c}}, \quad j \in\{\mathrm{II}, \mathrm{III}\}
\end{aligned}
$$

where overall void fraction of the bed $\varepsilon_{t}=\varepsilon_{c}+\varepsilon_{p}\left(1-\varepsilon_{c}\right)$, and $V_{c}$ denotes the volume of the chromatographic column. By scanning the points in the complete separation region of the $\left(m_{\mathrm{II}}, m_{\mathrm{III}}\right)$ plane, the optimal conditions can be located.

The complete separation region in the $\left(m_{\mathrm{II}}, m_{\mathrm{III}}\right)$ plane of the triangle theory was defined merely in terms of the purity performance indicator, that is, maximizing purities at the outlet streams with respect to the operating conditions $\theta$. In this study, the samples drawn from the MCMC algorithm are defined with respect to the multi-objective function (cf. Eq. (11)). All the collected samples (i.e., magenta triangle symbol in Fig. 5b) from the multivariate posterior distribution are plotted onto the $\left(m_{\mathrm{II}}, m_{\mathrm{III}}\right)$ plane. It is clearly shown that there is a strong linear correlation between the dimensionless ratios (the data is shown in D). As $m_{\mathrm{II}}$ and $m_{\mathrm{III}}$ are linearly correlated, so no samples are located inside the $\mathcal{D}$ region. In Fig. $5 \mathrm{~b}$, the samples that render $99 \%$ purities at both withdrawn outlets are illustrated with blue squares, while the $99.9 \%$ purities are illustrated with red dots. All these samples are located inside the complete separation region, $\mathcal{A}$, from the triangle theory. The black dot (i.e., $(0.308,0.484))$ on the $\left(m_{\mathrm{II}}, m_{\mathrm{III}}\right)$ plane is the optimum point from Klatt et al. (2002). It not located inside the ellipse of $99.9 \%$ purity samples, but in the ellipse of $99 \%$ samples. It is partially because of that the column length was optimized. Moreover, these samples are far away from both the vertex $(w)$, the diagonal $(x y)$ and the boundaries $(w x, w y)$ of the complete separation region. This also implies robustness of the operating conditions, as explained in detail in Mazzotti et al. (1997) that the 


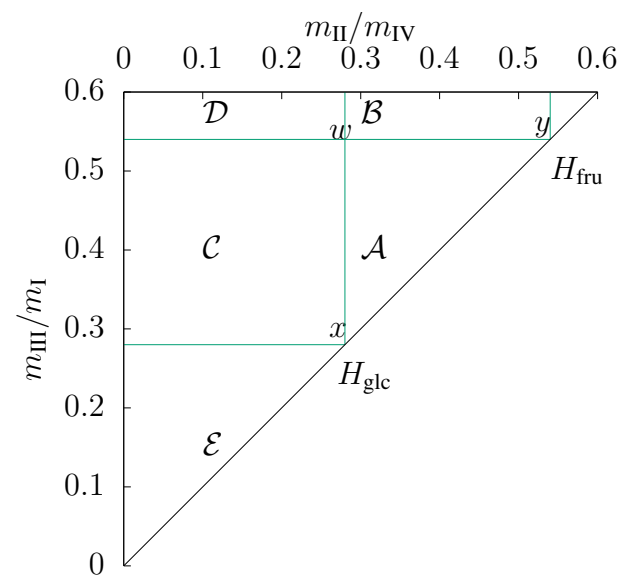

(a)

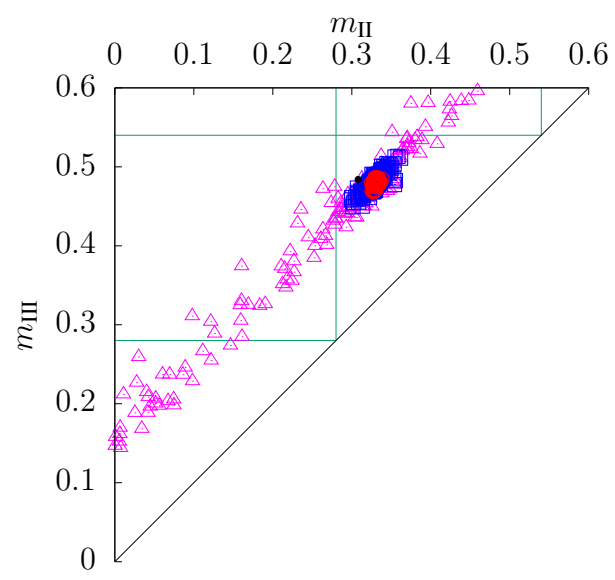

(b)

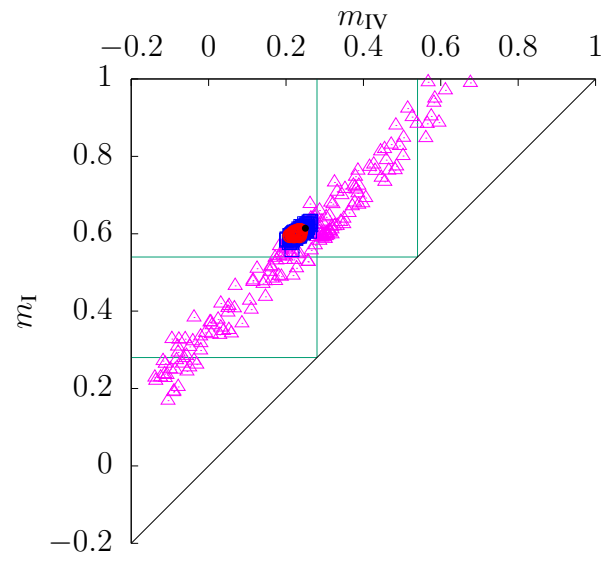

(c)

Figure 5: Schematic of the triangle theory $(\mathrm{a})$; the $\left(m_{\mathrm{II}}, m_{\mathrm{III}}\right)$ plane is divided into different separation regions; $w x, w y, x y$ shows the boundary of the complete separation region. The neither pure raffinate nor pure extract regions is denoted as $\mathcal{E}$. Bayesian based triangle theory of the $\left(m_{\mathrm{II}}, m_{\mathrm{III}}\right)$ plane $(\mathrm{b})$ and the $\left(m_{\mathrm{IV}}, m_{\mathrm{I}}\right)$ plane (c); the sample ensemble is illustrated with magenta triangles, while samples of $99 \%$ purities with blue squares and samples of $99.9 \%$ with red dots. 

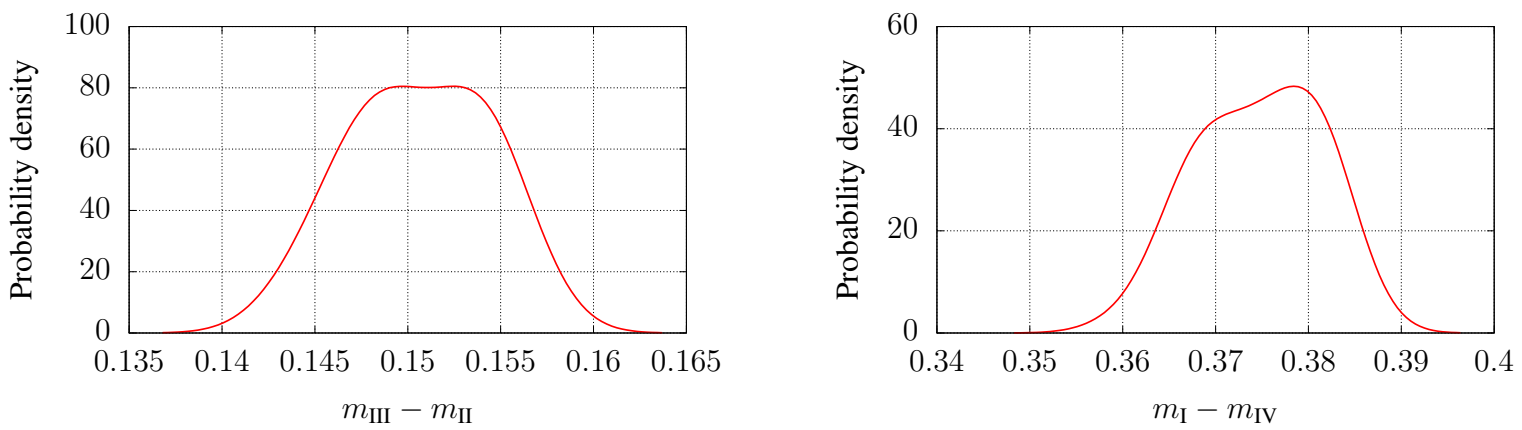

Figure 6: Probability distribution of the dimensionless ratio difference $m_{\mathrm{III}}-m_{\mathrm{II}}$ (left) and $m_{\mathrm{I}}-m_{\mathrm{IV}}$ (right).

minimal distance of the operating point from the boundaries of the complete separation region can be a measure of the maximal acceptance perturbation on the values of operating conditions. As seen from the sample density of different regions of the $\left(m_{\mathrm{II}}, m_{\mathrm{III}}\right)$ plane, operating points are more easily to be located in the $\mathcal{C}$ region than that in the $\mathcal{B}$ region. Therefore, from the SMB simulation point of view, it is more possible to encounter chromatograms upon CSS with pure raffinate streams and polluted extract streams (cf. the chromatogram of point $a$ in Fig. 2).

There is a cluster of optimal points merely in terms of the purity in the $\mathcal{A}$ region; they can be further distinguished according to other performance indicators. $m_{\mathrm{III}}-m_{\mathrm{II}}$ denotes the amount of fresh feed that is treated in the SMB process. The purity performance improves by increasing the $m_{\mathrm{III}}-m_{\mathrm{II}}$ difference, that is, moving the operating points towards the vertex, $w$, across straight lines parallel to the diagonal in the $\left(m_{\mathrm{II}}, m_{\mathrm{III}}\right)$ plane. On the contrary, the yield performance enhances by decreasing the $m_{\mathrm{III}}-m_{\mathrm{II}}$ difference; the pressure drops also limit the difference. As seen from Fig. 6, the $m_{\mathrm{III}}-m_{\mathrm{II}}$ that results in optimal conditions follows Gaussian distribution with mean value of 0.15 . It is located in the middle of the vertex, $w$, and the diagonal line, $x y$. Furthermore, it was observed that more numerical iterations were required to converge to CSS, when the operating points are close to boundaries of each region.

It is worth considering the $\left(m_{\mathrm{II}}, m_{\mathrm{III}}\right)$ plane of SMB units, which plays a key role in performing the separation. However, we also need to examine the projection of the four-dimensional region of the complete separation onto the $\left(m_{\mathrm{IV}}, m_{\mathrm{I}}\right)$ plane. A similar discussion applies to the $\left(m_{\mathrm{IV}}, m_{\mathrm{I}}\right)$ semi-infinite plane (i.e., $m_{\mathrm{IV}}<H_{\text {glc }}, H_{\text {fru }}<m_{\mathrm{I}}$ ) (cf. Fig. $5 \mathrm{c}$ ). The black dot $(0.250,0.614)$ is the optimum point from Klatt et al. (2002). Linear correlation is also observed, see D. The distribution of $m_{\mathrm{I}}-m_{\mathrm{IV}}$ is shown in Fig. 6; the value with the maximal probability is 0.378 . As observed in Fig. $5 \mathrm{c}$, the distance from the ellipse centre to the $w$ vertex (i.e., 0.09$)$ is shorter than that in the $\left(m_{\mathrm{III}}, m_{\mathrm{II}}\right)$ plane (i.e., 0.13). Thus, the ellipse is smaller and closer to the boundaries, in other words, less acceptance perturbation on the values of these operating conditions. If we consider the acceptance ratio of the MCMC sampling (the samples located in $\mathcal{D}$ over all samples), there is higher rejection rate in the $\left(m_{\mathrm{IV}}, m_{\mathrm{I}}\right)$ plane. It is also worth mentioning that the short distance from the ellipse centre to the vertex is partially due to the performance of desorbent consumption is not taken into account in the objective function.

As have discussed in the previous subsection, advantages of using Bayesian inference in uncertainty analysis is inherent. Advantages of Bayesian inference over the triangle theory in the determination of operating conditions is not obvious in this case study, where the linear isotherm is used and explicit algebraic expressions can be derived to calculate operating conditions. But, after having designed the complete separation region using the triangle theory, multiple operating points still need to be tested in order to pinpoint the optimal one in terms of performance indicators considered (Lübke et al., 2007; Heinonen et al., 2018). Moreover, in nonlinear cases (e.g., steric mass-action kinetics), the potential of Bayesian inference is prominent, where the complete separation region is strongly asymmetrical, curvilinear and the triangle theory even can not be applied. Nevertheless, the triangle theory is not trivial. It is most useful for the investigation of separation carried out under linear and quasi-linear conditions. Additionally, it provides a 
useful starting point for further nonlinear studies, as the condition of ideality and linearity can be relaxed.

\subsection{Multiple chain algorithm}

As have described in section 3, the multiple chain MCMC algorithm was not run intrinsically; instead, two simulation instances were run simultaneously on the Linux node, with an additional program checking the convergence periodically.

An inherent multiple chain MCMC algorithm, differential evolution Markov Chain (DE-MC (Braak, 2006; Vrugt et al., 2009)), has also been applied in this study. DE-MC is a combination of the differential evolution (Storn \& Price, 1997) with an added Metropolis step, in which multiple chains are run in parallel. Thus, it can be effective to explore multi-modal densities. 20 multiple chains were applied. The resulting posterior distributions of the operating conditions of the four-zone SMB process convey the same information (e.g., Fig. 1 and Fig. 3) as the ones from the presented MCMC algorithm (data is not shown). As have illustrated in Fig. 3, all the distributions are unimodal; therefore, both the MCMC and the DE-MC algorithms render the same results. Nevertheless, slightly differences of the smoothness of posterior distributions are observed, which is attributed to the use of different mechanism of proposal distributions. However, the performance and effectiveness of multiple chain MCMC algorithms could differ in process designs of SMB models with nonlinear adsorption isotherms or kinetics.

\section{Conclusions}

Any type of uncertainties both in the batch and the SMB chromatographic processes can affect their performance evaluation such that decision-making in application. Discrepancies between experimental and simulation results have commonly observed in SMB processes. Moreover, SMB industries have not been fully benefited from mechanistic model-based methods. These can often be attributed to the absence of uncertainty consideration. To this end, a Bayesian inference framework for the uncertainty assessment in the process design of SMB units has been introduced. A classical glucose-fructose four-zone SMB process under linear condition has been used as the example. A Markov Chain Monte Carlo algorithm, that is, Metropolis algorithm incorporated with delayed rejection and adjusted Metropolis strategies, has been used for sampling. The proposed method renders versatile information, e.g., well-determined operating conditions with credible intervals, robust posterior distributions, Pareto fronts and mapping to the triangle theory.

Although an SMB process with the adsorption behaviour described by the linear isotherm was used as an example, the power of proposed method is not limited to the linear situations. It is more powerful in the nonlinear scenarios. For instance, by using the Bayesian inference based methods, the separation regions of the triangle theory under strongly nonlinear conditions (where the triangle theory can not be applied) can be drawn. However, the computational cost, for cases with nonlinear kinetics and complex network configurations with a large number of columns, is expensive. The MCMC algorithm applied in this study is not implemented and coded in an intrinsic parallel manner. Though having compared the proposed MCMC algorithm to an inherent parallel algorithm leads to no different results of, such as, posterior distributions and credible intervals, the potential of multi-chain MCMC algorithms should be more prominent in cases of multi-modal target density.

\section{Acknowledgements}

This work is supported by the National Key Research and Development Program of China under Grant No. 2019YFD0901805 and 2017YFB0309302. 


\section{A Adaptive Metropolis}

A random draw from the proposal distribution of Metropolis algorithm with an initially given covariance $\Sigma_{0}=R_{0} R_{0}^{T}$ is

$$
\tilde{\theta}=\theta+R_{0} z
$$

where $z \in \mathbb{R}^{n}$ is an independent multivariate standard normal random vector. An initial covariance matrix $\Sigma_{0}$ is approximated with regard to the initial point, $\theta_{0}$, by using the Fisher information matrix:

$$
\begin{aligned}
\Sigma_{0} & \approx\left(\left(\frac{\partial c}{\partial \theta}\left(z=L, t, \theta_{0}\right)\right)^{T} \Lambda^{-1} \frac{\partial c}{\partial \theta}\left(z=L, t, \theta_{0}\right)\right)^{-1} \\
& =\tilde{\sigma}_{0}\left(\left(U S V^{T}\right)^{T}\left(U S V^{T}\right)\right)^{-1}=\tilde{\sigma}_{0}\left(V S^{T} U^{T} U S V^{T}\right)^{-1} \\
& =\tilde{\sigma}_{0}\left(V S^{T} S V^{T}\right)^{-1} \\
& =\tilde{\sigma}_{0} V \underbrace{\left(S^{T} S\right)^{-1}}_{=[\operatorname{diag}(S)]^{-2}} V^{T}
\end{aligned}
$$

Here, the weight matrix $\Lambda$ is $\operatorname{diag}\left(\tilde{\sigma}_{0}\right)$ and the Jacobian matrix $\frac{\partial c}{\partial \theta}\left(z=L, t, \theta_{0}\right)$ is calculated with automatic differentiation (Püttmann et al., 2016). It was then decomposed into $U S V^{T}$ using a singular value decomposition. The properties of $U^{T} U=I$ and $V^{-1}=V^{T}$ were used in the derivation of Eq. (29).

\section{B Delayed rejection}

The acceptance probability of the second stage candidate was computed in a way such that the reversibility of the Markov chain is preserved. Suppose the current position is $\theta^{i-1}$. A new candidate $\theta^{i}$, which was generated by the proposal distribution $J_{1}\left(\theta^{i} \mid \theta^{i-1}\right)$, is denied by the Metropolis probability. Upon rejection, a second stage candidate $\theta^{i+1}$ is tested, which depends not only on the current position $\theta^{i-1}$ but also the denied position $\theta^{i}$. The proposal is drawn from a different proposal distribution $J_{2}\left(\theta^{i+1} \mid \theta^{i-1}, \theta^{i}\right)$. The chance to accept the second stage candidate is determined by $\beta\left(\theta^{i-1}, \theta^{i}, \theta^{i+1}\right)=\min \left(1, \gamma\left(\theta^{i-1}, \theta^{i}, \theta^{i+1}\right)\right)$, where the ratio is

$$
\begin{aligned}
& \gamma\left(\theta^{i-1}, \theta^{i}, \theta^{i+1}\right) \\
& =\frac{p\left(\theta^{i+1} \mid \Psi\right) J_{1}\left(\theta^{i} \mid \theta^{i+1}\right) J_{2}\left(\theta^{i-1} \mid \theta^{i}, \theta^{i+1}\right)\left[1-\beta\left(\theta^{i}, \theta^{i+1}\right)\right]}{p\left(\theta^{i-1} \mid \Psi\right) J_{1}\left(\theta^{i} \mid \theta^{i-1}\right) J_{2}\left(\theta^{i+1} \mid \theta^{i}, \theta^{i-1}\right)\left[1-\beta\left(\theta^{i}, \theta^{i-1}\right)\right]} \\
& =\frac{p\left(\theta^{i+1} \mid \Psi\right) J_{1}\left(\theta^{i} \mid \theta^{i+1}\right)\left[1-\beta\left(\theta^{i}, \theta^{i+1}\right)\right]}{p\left(\theta^{i-1} \mid \Psi\right) J_{1}\left(\theta^{i} \mid \theta^{i-1}\right)\left[1-\beta\left(\theta^{i}, \theta^{i-1}\right)\right]} \\
& =q_{1} q_{2} \frac{1-\beta\left(\theta^{i}, \theta^{i+1}\right)}{1-\beta\left(\theta^{i}, \theta^{i-1}\right)}
\end{aligned}
$$

where a symmetric proposal distribution (i.e. $J_{2}$ ) was used as in the Metropolis algorithm. Therefore, $J_{2}$ is independent of the rejected position, $\theta^{i}$, such that $J_{2}\left(\theta^{i-1} \mid \theta^{i}, \theta^{i+1}\right)=J_{2}\left(\theta^{i+1} \mid \theta^{i}, \theta^{i-1}\right)$. The ratios $q_{1}$ and $q_{2}$ are given by

$$
\begin{aligned}
q_{1} & =\frac{p\left(\theta^{i+1} \mid \Psi\right)}{p\left(\theta^{i-1} \mid \Psi\right)} \\
& =\exp \left\{-\frac{1}{2}\left(\mathcal{H}\left(\theta^{i+1} ; d_{k}\right)-\mathcal{H}\left(\theta^{i-1} ; d_{k}\right)\right)\right\} \frac{p\left(\theta^{i+1}\right)}{p\left(\theta^{i-1}\right)}
\end{aligned}
$$


and

$$
\begin{aligned}
q_{2} & =\frac{J_{1}\left(\theta^{i} \mid \theta^{i+1}\right)}{J_{1}\left(\theta^{i} \mid \theta^{i-1}\right)} \\
& =\exp \left(-\frac{1}{2}\left[\left\|R^{-1}\left(\theta^{i+1}-\theta^{i}\right)\right\|^{2}-\left\|R^{-1}\left(\theta^{i-1}-\theta^{i}\right)\right\|^{2}\right]\right) .
\end{aligned}
$$

In Eq. (32), we have assumed a multivariate normal proposal density $J_{1}$ with covariance matrix $\Sigma=R R^{T}$.

\section{Autocorrelation plot of MCMC samples}

Autocorrelation, $\rho_{t}$, refers to the degree of correlation between the values of the same parameters across different observations (i.e., lag $t$ ). The pattern of autocorrelation of each parameter, $\theta_{\ell}$, is illustrated in Fig. 7. As seen from Fig. 7, when the lag $t>250$, the autocorrelation values $\rho_{t}$ get small enough. It can be interpreted as that, upon convergence, the samples generated from the stationary distribution are random and not correlated with each other.

\section{Linear correlations of $m_{j}$}

A linear correlation is observed between the dimensionless flowrate ratios, $m_{\mathrm{III}}$ and $m_{\mathrm{II}}$ (cf. Fig. 8). The top left and bottom right figures show the histograms of $m_{\mathrm{II}}$ and $m_{\mathrm{III}}$, respectively. The top right and bottom left figures both illustrate the linear correlation between $m_{\mathrm{III}}$ and $m_{\mathrm{II}}$. To be specific, $m_{\mathrm{III}}=1.0013 m_{\mathrm{II}}+0.1548$ and the coefficient determination of the fitting is $R^{2}=0.99$. In addition, the difference of $m_{\mathrm{III}}-m_{\mathrm{II}}$, which denotes the amount of fresh feed that is treated in the SMB process, is around 0.1548 . The most probable $m_{\mathrm{II}}$ value is 0.33 ; while the most probable $m_{\mathrm{III}}$ value is 0.48 . Therefore, the constraint $H_{\text {fru }}>m_{\mathrm{III}}>m_{\mathrm{II}}>H_{\text {glc }}$ holds.

$m_{\mathrm{I}}$ is also linearly correlated with $m_{\mathrm{IV}}, m_{\mathrm{I}}=0.9711 m_{\mathrm{IV}}+0.3605$, with the coefficient determination $R^{2}=0.98$. The difference of $m_{\mathrm{I}}-m_{\mathrm{IV}}$ denotes the amount of fresh desorbent that is treated in the SMB process. The most probable $m_{\mathrm{I}}$ value is 0.60 , while the most probable $m_{\mathrm{IV}}$ value is 0.23 . Thus, the constraints $H_{\text {fru }}<m_{\mathrm{I}}$ and $m_{\mathrm{IV}}<H_{\text {glc }}$ are satisfied.

\section{References}

Agrawal, G., \& Kawajiri, Y. (2012). Comparison of various ternary simulated moving bed separation schemes by multi-objective optimization. Journal of Chromatography A, 1238, 105-113.

Barber, J. A., Perkins, J. D., \& Sargent, R. W. (1998). Boundary conditions for flow with dispersion. Chemical Engineering Science, 53, 1463-1464.

Bentley, J., \& Kawajiri, Y. (2013). Prediction-correction method for optimization of simulated moving bed chromatography. AIChE Journal, 59, 736-746.

Bentley, J., Li, S., \& Kawajiri, Y. (2014). Experimental validation of optimized model-based startup acceleration strategies for simulated moving bed chromatography. Industrial $\mathscr{E}$ Engineering Chemistry Research, 53, 12063-12076.

Borg, N., Westerberg, K., Andersson, N., von Lieres, E., \& Nilsson, B. (2013). Effects of uncertainties in experimental conditions on the estimation of adsorption model parameters in preparative chromatography. Computers 83 Chemical Engineering, 55, 148-157.

Braak, C. J. T. (2006). A Markov Chain Monte Carlo version of the genetic algorithm Differential Evolution: easy Bayesian computing for real parameter spaces. Statistics and Computing, 16, 239-249. 


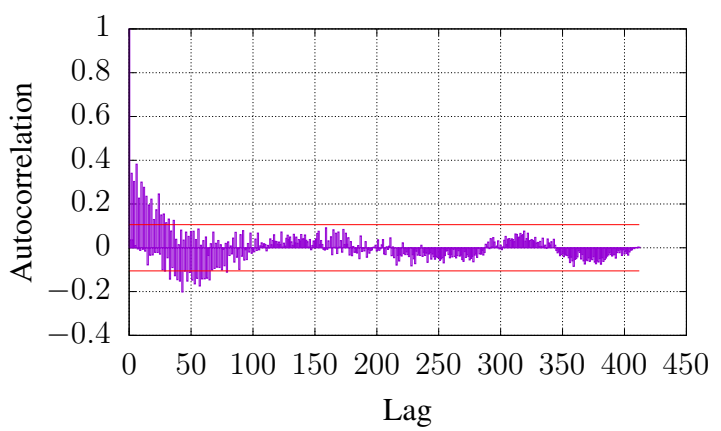

(a) $L$

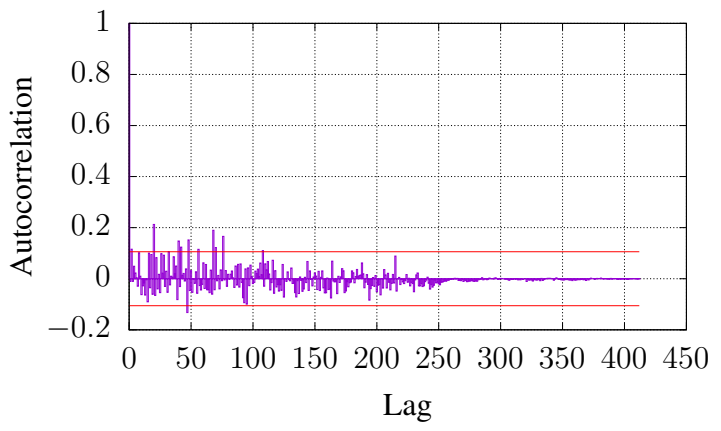

(c) $Q^{\text {rec }}$

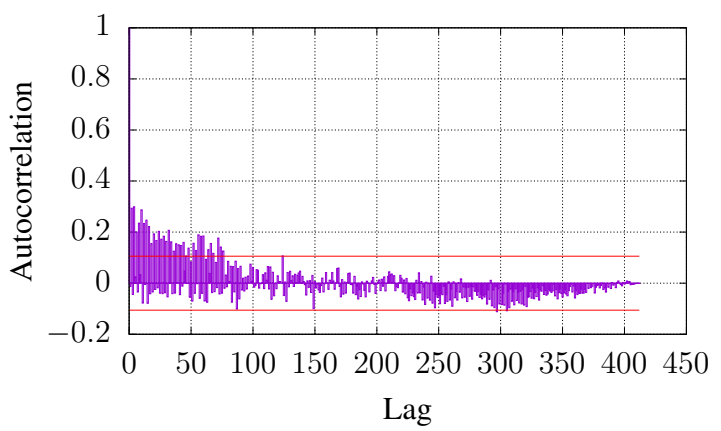

(e) $Q^{D}$

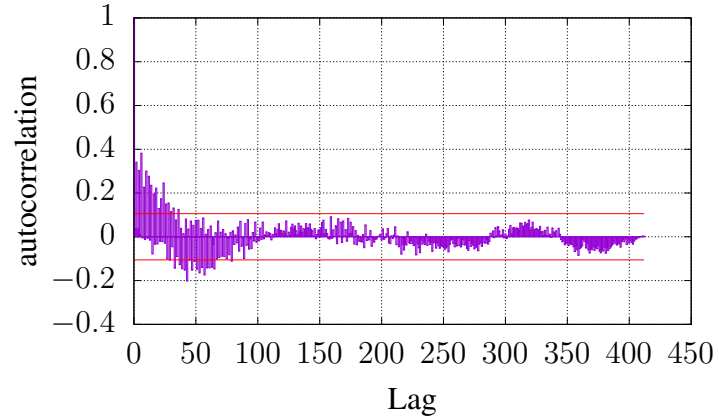

(b) $t_{s}$

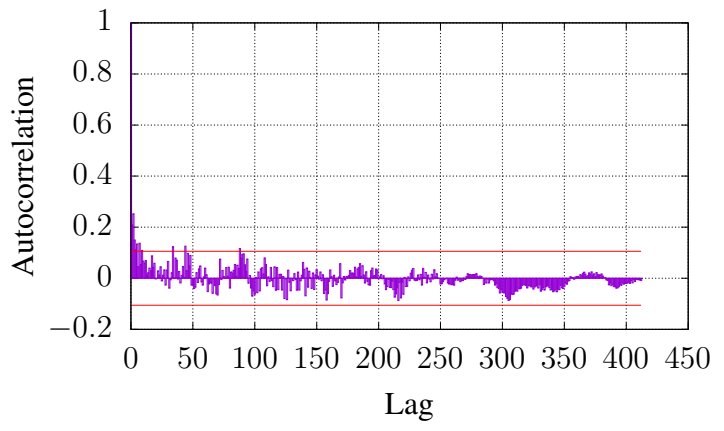

(d) $Q^{F}$

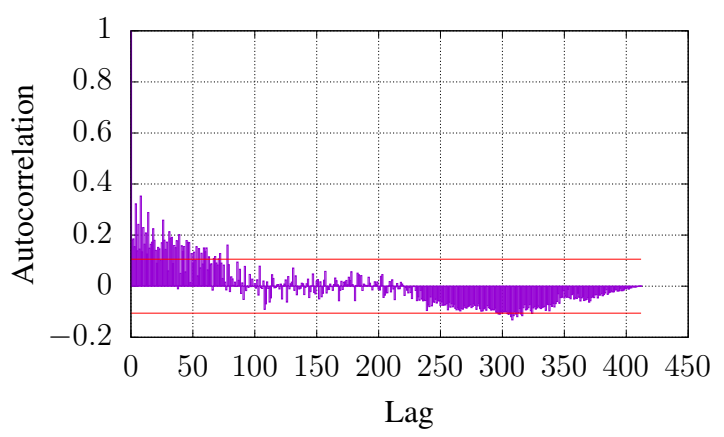

(f) $Q^{E}$

Figure 7: Autocorrelation plot of the operating conditions. 

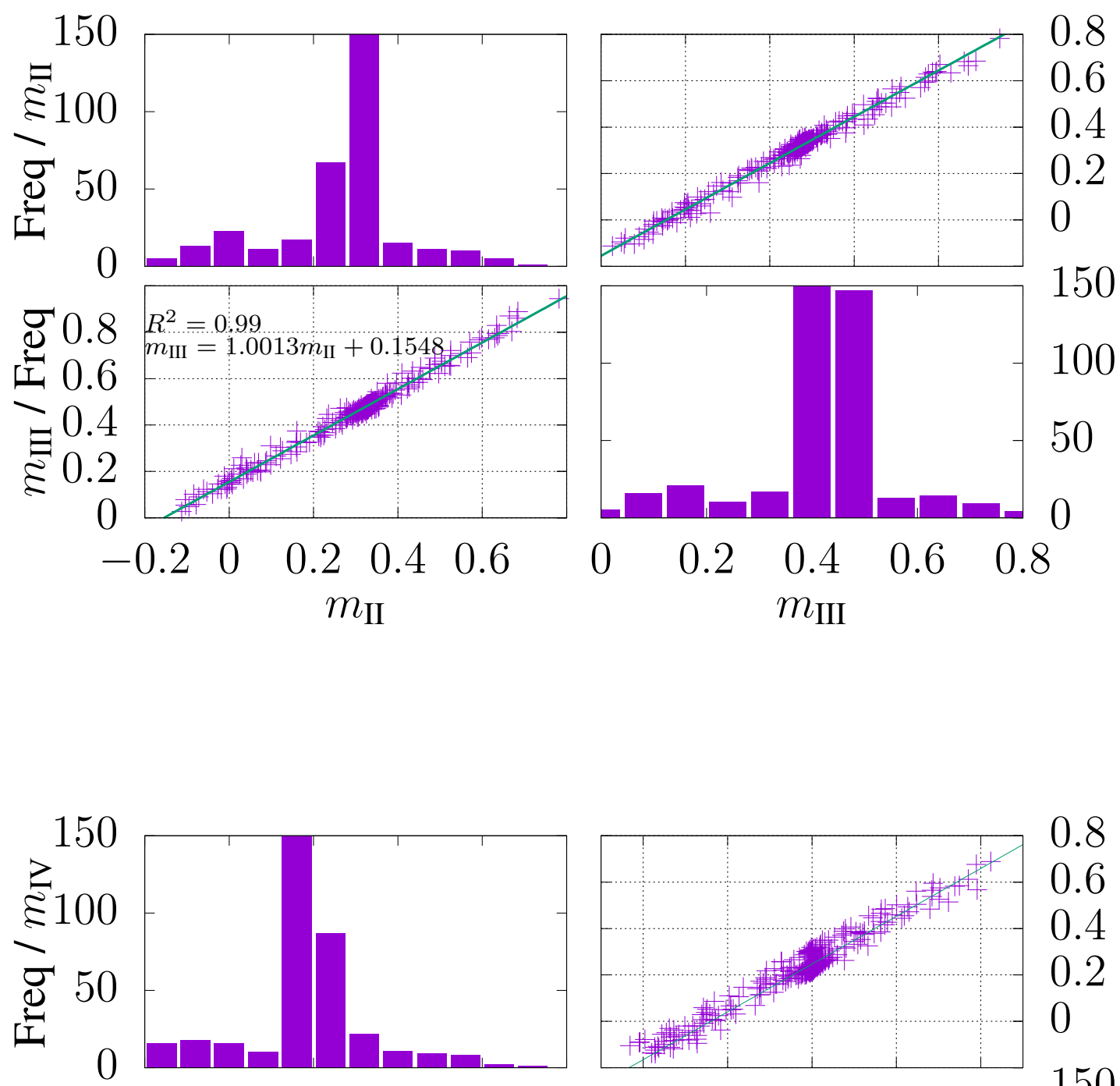

0.8

0.6

0.4

0.2

0
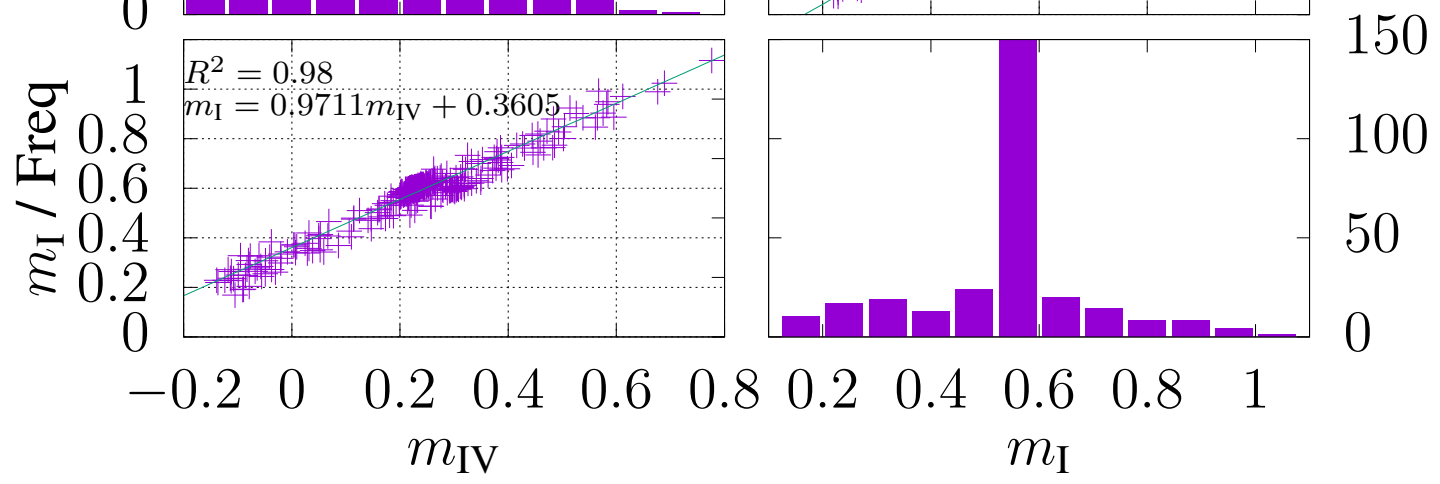

Figure 8: Linear correlation between the dimensionless values $m_{\mathrm{II}}, m_{\mathrm{III}}$ (top) and $m_{\mathrm{IV}}, m_{\mathrm{I}}$ (bottom). 
Briskot, T., Stückler, F., Wittkopp, F., Williams, C., Yang, J., Konrad, S., Doninger, K., Griesbach, J., Bennecke, M., Hepbildikler, S. et al. (2019). Prediction uncertainty assessment of chromatography models using Bayesian inference. Journal of Chromatography A, 1587, 101-110.

Brooks, C. A., \& Cramer, S. M. (1992). Steric mass-action ion exchange: displacement profiles and induced salt gradients. AIChE Journal, 38, 1969-1978.

Broughton, D. B., \& Gerhold, C. G. (1961). Continuous sorption process employing fixed bed of sorbent and moving inlets and outlets. US Patent 2,985,589.

Carta, G., \& Jungbauer, A. (2010). Protein chromatography: process development and scale-up. John Wiley \& Sons.

Chankong, V., \& Haimes, Y. Y. (2008). Multiobjective decision making: theory and methodology. Courier Dover Publications.

Clark, A. J., Kotlicki, A., Haynes, C. A., \& Whitehead, L. A. (2007). A new model of protein adsorption kinetics derived from simultaneous measurement of mass loading and changes in surface energy. Langmuir, 23, 5591-5600.

Diedrich, J., Heymann, W., Leweke, S., Hunt, S., Todd, R., Kunert, C., Johnson, W., \& von Lieres, E. (2017). Multi-state steric mass action model and case study on complex high loading behavior of mab on ion exchange tentacle resin. Journal of Chromatography A, 1525, 60-70.

Dismer, F., \& Hubbuch, J. (2010). 3D structure-based protein retention prediction for ion-exchange chromatogaphy. Journal of Chromatography A, 1217, 1343-1353.

Duh, K., Sudoh, K., Wu, X., Tsukada, H., \& Nagata, M. (2012). Learning to translate with multiple objectives. In Proceedings of the 50th Annual Meeting of the Association for Computational Linguistics (Volume 1: Long Papers) (pp. 1-10).

Dünnebier, G., Fricke, J., \& Klatt, K.-U. (2000). Optimal design and operation of simulated moving bed chromatographic reactors. Industrial \& engineering chemistry research, 39, 2290-2304.

Faria, R. P., \& Rodrigues, A. E. (2015). Instrumental aspects of simulated moving bed chromatography. Journal of Chromatography A, 1421, 82-102.

Gelman, A., Carlin, J. B., Stern, H. S., \& Rubin, D. B. (2014). Bayesian data analysis volume 2. Taylor \& Francis.

Ghosh, P., Lin, M., Vogel, J. H., Choy, D., Haynes, C., \& von Lieres, E. (2014). Zonal rate model for axial and radial flow membrane chromatography, part ii: Model-based scale-up. Biotechnology and bioengineering, $111,1587-1594$.

Ghosh, P., Vahedipour, K., Lin, M., Vogel, J. H., Haynes, C. A., \& von Lieres, E. (2013). Zonal rate model for axial and radial flow membrane chromatography. part i: Knowledge transfer across operating conditions and scales. Biotechnology and bioengineering, 110, 1129-1141.

Grosfils, V., Hanus, R., Wouwer, A. V., \& Kinnaert, M. (2010). Parametric uncertainties and influence of the dead volume representation in modelling simulated moving bed separation processes. Journal of Chromatography A, 1217, 7359-7371.

Guiochon, G. (2002). Preparative liquid chromatography. Journal of Chromatography A, 965, 129-161.

Guiochon, G., Felinger, A., \& Shirazi, D. G. (2006). Fundamentals of preparative and nonlinear chromatography. Academic Press. 
Haario, H., Laine, M., Mira, A., \& Saksman, E. (2006). DRAM: efficient adaptive MCMC. Statistics and Computing, 16, 339-354.

Heinonen, J., Laatikainen, M., \& Sainio, T. (2018). Chromatographic fractionation of a ternary mixture with an smb cascade process: The effect of ion exchange resin cross-linkage on separation efficiency. Separation and Purification Technology, 206, 286-296.

Joshi, M., Kremling, A., \& Seidel-Morgenstern, A. (2006). Model based statistical analysis of adsorption equilibrium data. Chemical Engineering Science, 61, 7805-7818.

Juza, M., Mazzotti, M., \& Morbidelli, M. (2000). Simulated moving-bed chromatography and its application to chirotechnology. Trends in biotechnology, 18, 108-118.

Kazi, M.-K., Medi, B., \& Amanullah, M. (2012). Optimization of an improved single-column chromatographic process for the separation of enantiomers. Journal of Chromatography A, 1231, 22-30.

Kim, K.-M., Song, J.-Y., \& Lee, C.-H. (2016). Combined operation of outlet streams swing with partial-feed in a simulated moving bed. Korean Journal of Chemical Engineering, 33, 1059-1069.

Kiwala, D., Mendrella, J., Antos, D., \& Seidel-Morgenstern, A. (2016). Center-cut separation of intermediately adsorbing target component by 8-zone simulated moving bed chromatography with internal recycle. Journal of Chromatography A, 1453, 19-33.

Klatt, K.-U., Hanisch, F., \& Dünnebier, G. (2002). Model-based control of a simulated moving bed chromatographic process for the separation of fructose and glucose. Journal of Process Control, 12, 203-219.

Kurup, A. S., Subramani, H. J., \& Harris, M. T. (2008). A Monte Carlo-based error propagation analysis of simulated moving bed systems. Separation and Purification Technology, 62, 582-589.

Lang, K. M., Kittelmann, J., Dürr, C., Osberghaus, A., \& Hubbuch, J. (2015). A comprehensive molecular dynamics approach to protein retention modeling in ion exchange chromatography. Journal of Chromatography A, 1381, 184-193.

Li, S., Feng, L., Benner, P., \& Seidel-Morgenstern, A. (2014a). Using surrogate models for efficient optimization of simulated moving bed chromatography. Computers $\&$ Chemical Engineering, 67, 121-132.

Li, S., Yue, Y., Feng, L., Benner, P., \& Seidel-Morgenstern, A. (2014b). Model reduction for linear simulated moving bed chromatography systems using krylov-subspace methods. AIChE Journal, 60, 3773-3783.

Liang, J., Fieg, G., Keil, F. J., \& Jakobtorweihen, S. (2012). Adsorption of proteins onto ion-exchange chromatographic media: A molecular dynamics study. Industrial \& Engineering Chmistry Research, 51, 16049-16058.

Lim, Y.-I. (2004). An optimization strategy for nonlinear simulated moving bed chromatography: multi-level optimization procedure (MLOP). Korean Journal of Chemical Engineering, 21, 836-852.

Lübke, R., Seidel-Morgenstern, A., \& Tobiska, L. (2007). Numerical method for accelerated calculation of cyclic steady state of ModiCon-SMB-processes. Computers \&3 Chemical Engineering, 31, 258-267.

Ma, Z., \& Wang, N.-H. (1997). Standing wave analysis of smb chromatography: linear systems. AIChE Journal, 43, 2488-2508.

Mallmann, T., Burris, B., Ma, Z., \& Wang, N. (1998). Standing wave design of nonlinear smb systems for fructose purification. AIChE journal, 44, 2628-2646.

Marler, R. T., \& Arora, J. S. (2010). The weighted sum method for multi-objective optimization: new insights. Structural and multidisciplinary optimization, 41, 853-862. 
Mavrotas, G. (2009). Effective implementation of the $\varepsilon$-constraint method in multi-objective mathematical programming problems. Applied mathematics and computation, 213, 455-465.

Mazzotti, M., Storti, G., \& Morbidelli, M. (1997). Optimal operation of simulated moving bed units for nonlinear chromatographic separations. Journal of Chromatography A, 769, 3-24.

Metropolis, N., Rosenbluth, A. W., Rosenbluth, M. N., Teller, A. H., \& Teller, E. (1953). Equation of state calculations by fast computing machines. The Journal of Chemical Physics, 21, 1087-1092.

Mihlbachler, K., Fricke, J., Yun, T., Seidel-Morgenstern, A., Schmidt-Traub, H., \& Guiochon, G. (2001). Effect of the homogeneity of the column set on the performance of a simulated moving bed unit: I. theory. Journal of Chromatography A, 908, 49-70.

Nowak, J., Antos, D., \& Seidel-Morgenstern, A. (2012). Theoretical study of using simulated moving bed chromatography to separate intermediately eluting target compounds. Journal of Chromatography A, 1253, 58-70.

Püttmann, A., Schnittert, S., Leweke, S., \& von Lieres, E. (2016). Utilizing algorithmic differentiation to efficiently compute chromatograms and parameter sensitivities. Chemical Engineering Science, 139, $152-162$.

Rajendran, A., Paredes, G., \& Mazzotti, M. (2009). Simulated moving bed chromatography for the separation of enantiomers. Journal of Chromatography A, 1216, 709-738.

Rodrigues, R. C., Araújo, J. M., Eusébio, M. F., \& Mota, J. P. (2007). Experimental assessment of simulated moving bed and varicol processes using a single-column setup. Journal of Chromatography A, 1142, 69-80.

Ruthven, D. M., \& Ching, C. (1989). Counter-current and simulated counter-current adsorption separation processes. Chemical Engineering Science, 44, 1011-1038.

Samuelsson, J., Sajonz, P., \& Fornstedt, T. (2008a). Impact of an error in the column hold-up time for correct adsorption isotherm determination in chromatography: I. even a small error can lead to a misunderstanding of the retention mechanism. Journal of Chromatography A, 1189, 19-31.

Samuelsson, J., Zang, J., Murunga, A., Fornstedt, T., \& Sajonz, P. (2008b). Impact of an error in the column hold-up time for correct adsorption isotherm determination in chromatography: Ii. can a wrong column porosity lead to a correct prediction of overloaded elution profiles? Journal of Chromatography A, 1194, $205-212$.

Scopes, R. K. (2013). Protein purification: principles and practice. Springer Science \& Business Media.

Seidel-Morgenstern, A., Kessler, L. C., \& Kaspereit, M. (2008). New developments in simulated moving bed chromatography. Chemical Engineering \& Technology, 31, 826-837.

Silva, M. S., Rodrigues, A. E., \& Mota, J. P. (2015). Modeling and simulation of an industrial-scale parex process. AIChE Journal, 61, 1345-1363.

Sreedhar, B., Hobbs, D. T., \& Kawajiri, Y. (2014). Simulated moving bed chromatography designs for lanthanide and actinide separations using Reillex $\mathrm{HPQ}^{\mathrm{TM}}$ resin. Separation and Purification Technology, $136,50-57$.

Storn, R., \& Price, K. (1997). Differential evolution-a simple and efficient heuristic for global optimization over continuous spaces. Journal of Global Optimization, 11, 341-359.

Storti, G., Baciocchi, R., Mazzotti, M., \& Morbidelli, M. (1995). Design of optimal operating conditions of simulated moving bed adsorptive separation units. Industrial 85 engineering chemistry research, 34 , $288-301$. 
Storti, G., Mazzotti, M., Morbidelli, M., \& Carrà, S. (1993). Robust design of binary countercurrent adsorption separation processes. AIChE Journal, 39, 471-492.

Toumi, A., Engell, S., Diehl, M., Bock, H. G., \& Schlöder, J. (2007). Efficient optimization of simulated moving bed processes. Chemical Engineering and Processing: Process Intensification, 46, 1067-1084.

Vrugt, J. A., Ter Braak, C., Diks, C., Robinson, B. A., Hyman, J. M., \& Higdon, D. (2009). Accelerating Markov Chain Monte Carlo simulation by differential evolution with self-adaptive randomized subspace sampling. International Journal of Nonlinear Sciences and Numerical Simulation, 10, 273-290.

Wu, X., Arellano-Garcia, H., Hong, W., \& Wozny, G. (2013). Improving the operating conditions of gradient ion-exchange simulated moving bed for protein separation. Industrial $\&$ Engineering Chemistry Research, 52, 5407-5417.

Yao, C., Tang, S., Lu, Y., Yao, H.-M., \& Tade, M. O. (2015). Combination of space-time conservation element/solution element method and continuous prediction technique for accelerated simulation of simulated moving bed chromatography. Chemical Engineering and Processing: Process Intensification, 96, $54-61$.

Yu, Y., Wood, K. R., \& Liu, Y. (2015). Simulation and comparison of operational modes in simulated moving bed chromatography. Industrial \& Engineering Chemistry Research, 54, 11576-11591.

Zhang, L., Selker, J., Qu, A., \& Velayudhan, A. (2001). Numerical estimation of multicomponent adsorption isotherms in preparative chromatography: implications of experimental error. Journal of Chromatography A, 934, 13-29.

Zhang, Z., Hidajat, K., Ray, A. K., \& Morbidelli, M. (2002). Multiobjective optimization of smb and varicol process for chiral separation. AIChE Journal, 48, 2800-2816. 